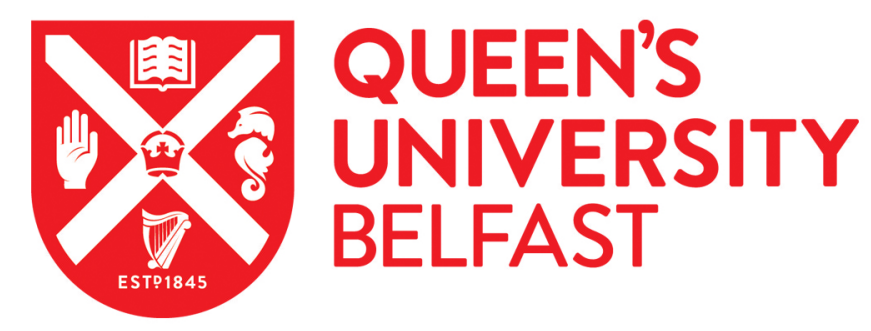

\title{
Delivery of RALA/siFKBPL nanoparticles via electrospun bilayer nanofibres: An innovative angiogenic therapy for wound repair
}

Mulholland, E., Ali, A., Robson, T., Dunne, N., \& McCarthy, H. (2019). Delivery of RALA/siFKBPL nanoparticles via electrospun bilayer nanofibres: An innovative angiogenic therapy for wound repair. Journal of Controlled Release, 316, 53-65. https://doi.org/10.1016/j.jconrel.2019.10.050

Published in:

Journal of Controlled Release

Document Version:

Peer reviewed version

Queen's University Belfast - Research Portal:

Link to publication record in Queen's University Belfast Research Portal

\section{Publisher rights}

Copyright 2019 Elsevier.

This manuscript is distributed under a Creative Commons Attribution-NonCommercial-NoDerivs License

(https://creativecommons.org/licenses/by-nc-nd/4.0/), which permits distribution and reproduction for non-commercial purposes, provided the author and source are cited.

\section{General rights}

Copyright for the publications made accessible via the Queen's University Belfast Research Portal is retained by the author(s) and / or other copyright owners and it is a condition of accessing these publications that users recognise and abide by the legal requirements associated with these rights.

\section{Take down policy}

The Research Portal is Queen's institutional repository that provides access to Queen's research output. Every effort has been made to ensure that content in the Research Portal does not infringe any person's rights, or applicable UK laws. If you discover content in the Research Portal that you believe breaches copyright or violates any law, please contact openaccess@qub.ac.uk. 


\section{Delivery of RALA/siFKBPL}

\section{Nanoparticles via Electrospun}

\section{Bilayer Nanofibres: an innovative}

\section{angiogenic therapy for wound repair}

\section{Authors}

Eoghan J. Mulholland ${ }^{1}$, Ahlam Ali², Tracy Robson ${ }^{3}$, Nicholas J. Dunne ${ }^{2,}$ 4-8, Helen O. McCarthy $^{2}$.

${ }^{1}$ Gastrointestinal Stem Cell Biology Laboratory, Wellcome Trust Centre for Human Genetics, University of Oxford, Oxford, United Kingdom, OX3 7BN

${ }^{2}$ School of Pharmacy, Queen's University Belfast, 97 Lisburn Road, Belfast BT9 7BL, UK.

${ }^{3}$ Molecular \& Cellular Therapeutics, Royal College of Surgeons in Ireland, Dublin, Ireland.

${ }^{4}$ Centre for Medical Engineering Research, School of Mechanical and Manufacturing Engineering, Dublin City University, Stokes Building, Collins Avenue, Dublin 9, Ireland.

${ }^{5}$ School of Mechanical and Manufacturing Engineering, Dublin City University, Stokes Building, Collins Avenue, Dublin 9, Ireland.

${ }^{6}$ Trinity Centre for Bioengineering, Trinity Biomedical Sciences Institute, Trinity College Dublin, Dublin 2, Ireland.

${ }^{7}$ Department of Mechanical and Manufacturing Engineering, School of Engineering, Trinity College Dublin, Dublin, Ireland.

${ }^{8}$ Advanced Materials and Bioengineering Research Centre (AMBER), Royal College of Surgeons in Ireland and Trinity College Dublin, Dublin, Ireland. 


\section{Abstract}

FK506-binding protein-like (FKBPL) has previously been shown to inhibit angiogenesis via in vitro and in vivo experimentation. Thus, it was proposed that the delivery of a siRNA targeting FKBPL could hold great potential in promoting angiogenesis for advanced wound healing applications. An effective delivery system has been utilised to encapsulate the siFKBPL to form nanoparticles, thereby improving cellular entry and eliciting a potent angiogenic response. In this study, nanoparticles were formed via condensation of siFKBPL with RALA; a novel, cationic 30 mer amphipathic peptide. Nanoparticles prepared at a N:P ratio of 6 demonstrated an average particle size of $76.6 \mathrm{~nm}$ with a zeta potential of $+16.5 \mathrm{mV}$. Treatment of HMEC-1 cells at N:P 6 resulted in a transfection efficiency of 33.7\%, negligible cytotoxicity, and significant knockdown of endogenous FKBPL expression. Functionally, treatment with RALA/siFKBPL resulted in significant improvements in cell migration and endothelial tubule formation in vitro. The process of electrospinning was employed to fabricate a nanofibrous wound patch to facilitate the controlled delivery of the RALA/siFKBPL nanoparticles. Alginate/poly-(vinyl alcohol) was electrospun following electrospinning of Chitosan/poly-(vinyl alcohol) to form a bilayered wound patch. Subsequently, the nanofibres were crosslinked to improve stability, before nanoparticle incorporation via soak loading. In vivo wound healing studies using C57BL/6J mice demonstrated a significant increase in angiogenesis when the RALA/siFKBPL nanoparticles were delivered from the bilayered wound patch; a $326 \%$ increase in blood vessel density was observed compared to untreated wounds. Taken together, this data demonstrates that delivery of RALA/siFKBPL nanoparticles from the bilayered wound patch represents an innovative wound healing therapy.

Key Words: Angiogenesis, Wound Healing, Nanoparticles, siRNA, Gene Therapy, Electrospinning 


\section{Introduction}

The process of wound healing is one of the most complex biological mechanisms; it is highly coordinated and involves three overlapping phases: (i) Inflammation, (ii) Proliferation, and (iii) Remodelling [1]. Failure within any of these phases can result in a delayed healing process and subsequent development of a chronic wound. Local influences such as infection or oedema directly affect the characteristics of the wound site and negatively impact wound repair. Systemic influences such as the general overall health, disease state or comorbidities of the patient can also delay repair [2].

Delayed wound healing can be linked to a dysregulation in genes, which regulate processes such as angiogenesis. Blood vessel establishment is an essential component for wound healing as the demand for oxygen and nutrients is high. Local conditions such as a low $\mathrm{pH}$ or increased lactate levels in the microenvironment of the wound initiate the release of angiogenic factors. These factors include vascular endothelial growth factor (VEGF), basic fibroblast growth factor (bFGF), and transforming growth factor-beta (TGF- $\beta$ ) [3]. Such factors are produced by epidermal macrophages, fibroblasts and vascular endothelial cells [4]. Endothelial cells express fibronectin receptors and proteases required to advance past the granulation tissue. These activated cells form buds from neighbouring capillary vessels. In time these buds will coalesce and canalise to form neo-capillaries throughout the granulation tissue, consequently providing blood flow to the area [3]. Indeed, patients suffering from diabetes, for example, encounter hyperglycaemia, which leads to the stiffening of vessel walls, resulting in a reduction in blood flow. This reduction in blood flow deprives the wound site of much-needed oxygen and nutrients [5]. As a result, hypoxia is prevalent in diabetic wounds, and this inhibits the migration of endothelial cells thereby reducing angiogenesis [6]. 
Therapeutic angiogenesis is therefore a promising concept for the treatment of such wounds. FK506-binding protein-like (FKBPL) is a member of the FK506 binding proteins family and is a potent antiangiogenic protein which targets the CD44 pathway, and so inhibition offers a potential angiogenic therapy [7]. Promisingly, a therapeutic peptide based on the antiangiogenic domain of the FKBPL gene termed ALM201 has now entered Phase I/II clinical trials (EudraCT number: 2014-001175-31).

RALA was developed by McCarthy et al. (2014) and is composed of a 30 amino acid sequence derived from KALA by the exchange of lysine groups in favour of arginine. RALA has a welldocumented history of successfully delivering anionic therapeutics (e.g. plasmids [8-11], bisphosphonates [12], and siRNAs [9]). In a previous study, Bennett et al. complexed RALA with both plasmid encoding FKBPL (pFKBPL) and siRNA targeting FKBPL (siFKBPL). The authors reported the effect of each treatment on angiogenesis in vivo in an MDA-MB-231 breast cancer xenograft model using SCID mice. Histological analysis revealed that a significant increase in the number of blood vessels (38\%) was observed in tumours treated with RALA/siFKBPL. Moreover, a reduction of 53\% in vessel number was observed in tumours treated with RALA/pFKBPL [9].

The electrospinning of polymers to produce nanofibres has received much attention; this is mainly due to the ease of set-up and fibre production, in addition to low-cost [13-15]. Differences in voltage between injection needles and ground collectors drive the charged polymer to form jets at the tip of the injection needles; this is known as the 'Taylor cone' effect. Electrospun nanofibres hold great potential as biomaterials in wound healing applications. These two-dimensional, non-woven structures have been shown to enable fluid absorption with 
excellent gas permeation properties, which facilitates cell respiration when applied to open wounds [16-17].

The poly-(vinyl alcohol) (PVA) polymer is FDA approved and has a proven track record in the biomedical industry for applications such as contact lenses, artificial pancreas, and synthetic vitreous humor [18-20]. PVA is an attractive option as it is biocompatible with low protein adsorption properties. This results in a low affinity for adhesion to cells, which for a wound dressing is critical to avoid disruption of the tissue upon removal [21]. Alginate (ALG) is a natural polymer with excellent biocompatibility that is sourced from seaweeds $[22,23]$, and is ideal for wound dressings as it accommodates a moist microenvironment and can accelerate re-epithelisation [24]. Chitosan (CHT) is another natural polymer derived from Chitin, which can be obtained from the shells of crustaceans [25]. As a semipermeable natural polymer, chitosan maintains a sterile condition by allowing efficient gas and water vapor exchange, while protecting against microorganism permeation into the wound [25-26].

Herein, we investigated the delivery of siFKBPL nanocomplexed with the RALA peptide as a novel gene therapy approach to reduce endogenous FKBPL levels and promote angiogenesis for wound healing benefits. The RALA/siFKBPL complexes were incorporated into an electrospun nanofibrous wound patch composed of ALG, CHT and PVA for in vitro and in vivo assessment. The therapy was tested in vitro using HMEC-1 endothelial cells and then assessed under in vivo conditions using a full-thickness skin wounding model in C57BL/6J mice. 


\section{Materials and Methods}

\section{Materials}

\section{RALA peptide}

The RALA peptide (Biomatik, USA) was produced by solid-state synthesis, and supplied as a lyophilised powder, with the purity defined as desalted. The 30 amino acid sequence of RALA is:

\section{N-WEARLARALARALARHLARALARALRACEA-C}

\section{Nucleic Acids}

Human siFKBPL, murine siFKBPL and scrambled siRNA (siSC) (Dharmacon, UK) were supplied as a lyophilised powder containing $20 \mathrm{nM}$ of oligonucleotides. Human siFKBPL was used for in vitro analysis of FKBPL knockdown, and murine siFKBPL was used for in vivo experimentation. pEGFP-N1 was purchased from Clontech (USA). The plasmid was propagated in MAX Efficiency ${ }^{\circledR}$ DH5 $\alpha^{\mathrm{TM}}$ Competent Cells (Life Technologies, UK), purified using PureLink ${ }^{\circledR}$ HiPure Plasmid Filter Maxiprep Kit (Life Technologies, UK), and quantified by UV absorption at $260 \mathrm{~nm}$.

\section{Cell lines}

The immortalised human microvascular endothelial cell line (HMEC-1) was obtained from American Type Culture Collection (USA) and maintained in MCDB-131 medium (Thermo Fisher, USA) supplemented with 10\% fetal calf serum (FCS) (Thermo Fisher, USA) epidermal growth factor (EGF, $10 \mathrm{ng} / \mathrm{ml})$ (Roche, Switzerland) and L-glutamine $(10 \mathrm{mM})$ (Thermo Fisher, USA). The cell line was authenticated by short tandem repeat (STR) profiling completed by the suppliers and were routinely verified to be Mycoplasma free. All experiments were conducted at $37^{\circ} \mathrm{C}$ in a humidified atmosphere of $5 \% \mathrm{CO}_{2} / 95 \% \mathrm{O}_{2}$. 


\section{Polymers}

Poly-(vinyl alcohol) $(\mathrm{PVA})(\mathrm{MW}=146-186 \mathrm{kDa})$, sodium alginate $(\mathrm{ALG})(\mathrm{MW}=120-190$ $\mathrm{kDa})$ and chitosan $(\mathrm{CHT})(\mathrm{MW}=50-190 \mathrm{kDa})$ were purchased from Sigma-Aldrich, UK.

\section{Animals}

Six-week-old C57BL/6J mice were purchased from Charles River Laboratories (UK). The animals were housed in an open facility at $21^{\circ} \mathrm{C}$ and $50 \%$ humidity with food and water ad libitum. The experimental protocols were compliant with the UK Scientific Act of 1986 and performed under project license 2678.

\section{Methods}

\section{Synthesis of RALA/siFKBPL and RALA/pEGFP-N1 Nanoparticles}

RALA-pDNA and RALA-siRNA nanoparticles were prepared at N:P ratios $0-12$ by adding appropriate volumes of RALA peptide solution to $1 \mu \mathrm{g}$ siRNA or pDNA as detailed in Bennett et al. (2015) [9].

\section{Nanoparticle Size and Zeta Potential Analysis}

It is essential to characterise nanoparticle systems in terms of size and charge as these parameters influence cellular internalisation rate. The mean hydrodynamic size of the complexes was determined using Dynamic Light Scattering on a Malvern Nano ZS (Malvern Instruments, UK). Mean surface charge (zeta potential) of the complexes was also measured by using Laser Doppler Velocimetry on a Malvern Nano ZS. 


\section{Stability of RALA/siFKBPL Nanoparticles over a Range of Temperatures}

The stability of nanoparticles over a range of temperatures was conducted to determine the impact on the physicochemical characteristics. Nanoparticles were prepared at N:P 6 and added to a polystyrene cuvette (Sarstedt, UK). A N:P ratio of 6 was found to be optimal for HMEC1 cell transfection. Stability was assessed via hydrodynamic size analysis over a range of temperatures $\left(4-37^{\circ} \mathrm{C}\right)$ using the Nano ZS DTS software (Malvern Instruments, UK).

\section{Transmission Electron Microscopy (TEM) of RALA/siFKBPL Nanoparticles}

Visualisation of the nanoparticles using TEM is important as it allows for the confirmation of nanoparticle size data as collected using dynamic light scattering. RALA/siFKBPL complexes were prepared at an $\mathrm{N}: \mathrm{P}$ ratio of 6 containing $1 \mu \mathrm{g}$ of siFKBPL and a Copper/Carbon mesh grid (TAAB Laboratories, UK) placed face down on a $10 \mu \mathrm{L}$ sample of the complexes for 1 min. The loaded grid was dried overnight and stained for $30 \mathrm{~s}$ with UranyLess (EMS, USA), a stain for ultrafine section, at room temperature. The grid was imaged using a JEM-1400 Plus Transmission Electron Microscope (JEOL, USA) at an accelerating voltage of $80 \mathrm{kV}$.

\section{Gel Electrophoresis of RALA/siFKBPL Nanoparticles}

Gel electrophoresis of RALA/siFKBPL nanoparticles over a range of N:P ratios allowed for the visualisation of the extent of complexation, which is important in the development of a nanoparticle device to ensure there is minimal free siRNA. Nanoparticles were prepared and allowed to stand for $30 \mathrm{~min}$ at room temperature $\left(22^{\circ} \mathrm{C} \pm 0.5^{\circ} \mathrm{C}\right)$. Electrophoresis sample loading dye (Bio-RAD, UK) was added, and samples were loaded into the wells of a $1 \% \mathrm{w} / \mathrm{v}$ agarose gel (Bioline, UK) containing $0.2 \mu \mathrm{g} / \mathrm{mL}$ ethidium bromide (Sigma-Aldrich, UK). Gels were run at $100 \mathrm{~V}$ for $60 \mathrm{~min}$ and subsequently visualised under $\mathrm{UV}$ light using a Multispectrum Bioimaging System (UVP, UK). 


\section{Transfection studies in HMEC-1 cells in vitro}

HMEC-1 cells were seeded at a density of $2 \times 10^{4}$ and $3 \times 10^{4}$ cells per well in 96-well and 24 well plates (VWR, UK) and left to adhere for $24 \mathrm{~h}$ prior to transfection. Thereafter, the media was removed from each well and replaced with $100 \mu \mathrm{L}$ (96 well plate) or $200 \mu \mathrm{L}$ (24 well plate) of Opti-MEM media (Thermo Fisher, USA) and left for a further $2 \mathrm{~h}$. Each well was then supplemented with RALA transfection complexes for $4 \mathrm{~h}$, after which the well was aspirated prior to the addition of normal expansion media.

\section{Cell Viability Analysis of HMEC-1 cells subject to Transfection}

Cell viability studies are important in the development of a wound healing device to ensure no compromise is made to the already delicate tissue. HMEC-1 endothelial cells were seeded in 96-well plates (VWR, UK) at a density of $10 \times 10^{3}$ cells per well and transfected as described above and allowed to incubate at $37^{\circ} \mathrm{C}$ under $5 \% \mathrm{CO}_{2}$ for $24 \mathrm{~h}$. MTS reagent (Promega, USA) was then added to the media $24 \mathrm{~h}$ post-transfection at a concentration of $10 \%$ and incubated under the dark conditions for a further $2 \mathrm{~h}$. Absorbance was then recorded at $490 \mathrm{~nm}$ using an OmniStar plate reader (BioTek Instruments Inc., UK). Cell viability was presented as a percentage relative to untreated cells control.

\section{Western Blot Analysis of Endogenous FKBPL Levels in Transfected HMEC-1 cells}

Western blot analysis of protein levels is important in the development of the nanoparticle systems to ensure the functionality of the siRNA transfection. Transfection was conducted for $48 \mathrm{~h}$, and cell lysates were harvested by aspiration of the media, followed by the addition of Laemmli $2 \times$ sample buffer (Sigma Aldrich, UK). Cell lysates were subjected to SDS-PAGE electrophoresis using a NuPAGE ${ }^{\circledR} 4-12 \%$ Bis-Tris gel (Life Technologies, UK) and the XCellSurelock $^{\mathrm{TM}}$ electrophoresis cell (Invitrogen, UK), transferred to nitrocellulose membranes, blocked for $1 \mathrm{~h}$ at room temperature in 3\% skimmed milk powder (Merck, UK) 
blocking solution in PBS-Tween. The membrane was then probed with 1:1000 dilution of rabbit FKBPL IgG polyclonal primary antibody (Proteintech, UK), 1:5000 dilution of rabbit GAPDH primary antibody (Sigma-Aldrich, UK) and 1:5000 dilution of ECL anti-rabbit IgG HRP-linked whole secondary antibody (GE Healthcare UK Ltd, UK). Antibody binding was detected using Immobilon ${ }^{\mathrm{TM}}$ Western chemiluminescent HRP substrate (Millipore, UK) and imaged using a G-box imaging system (Syngene, UK). Densitometric analysis was performed, and the FKBPL was quantified relative to GAPDH using ImageJ software (version 1.52a, NIH, USA).

\section{Wound Scratch Assay with Transfected HMEC-1 cells}

The wound scratch assay is a means of measuring the effects of RALA/siFKBPL on cell migration. With endothelial cell migration being compromised within the delayed wound healing environment, it is important to understand the impact of RALA/siFKBPL on it. Wound scratch culture inserts (Ibidi, UK) were directly pipetted with $70 \times 10^{3}$ cells in a 24 -well plate. Culture-inserts were gently removed using sterile tweezers $12 \mathrm{~h}$ post-seeding, and the cell layer was washed using PBS ( $\mathrm{pH}=7.4)$. Then $1 \mathrm{~mL}$ of cell culture medium $(2 \%$ FCS $)$ was placed in each well of the 24-well plate. Cells were imaged using an EVOS FL Cell Imaging System (Life Technologies, UK) at different time points up to $24 \mathrm{~h}$. The remaining wound area was presented as percentage closure from $0 \mathrm{~h}$.

\section{Tubule Formation Assay with Transfected HMEC-1 cells}

The tubule formation assay is a means of measuring the effects of treatment on the reorganisation stage of angiogenesis. With angiogenesis being compromised in a delayed wound healing environment, it is important to demonstrate the effects of RALA/siFKBPL at the wound site. Matrigel (Corning, UK) was pipetted into a 96-well plate at a volume of $70 \mu \mathrm{L}$ and left to incubate for $1 \mathrm{~h}$ at $37^{\circ} \mathrm{C}$. HMEC-1 cells were subject to transfection and trypsinised 
before being suspended in serum-free media. Cells were counted, and $20 \times 10^{3}$ cells were pipetted onto the Matrigel. Cells were left overnight for $14 \mathrm{~h}$ to allow sufficient time for tubules to form. The extent of tubule formation was quantified using WimTube software online (https://www.wimasis.com/en/products/13/WimTube) with images taken using an EVOS FL Cell Imaging System (Life Technologies) once tubules had established and imaged up to $24 \mathrm{~h}$. Results were represented as the total tubule number compared to the untreated control at $0 \mathrm{~h}$.

\section{Fabrication of Bilayered Electrospun Nanofibre Wound Patch}

The bilayered wound patch was fabricated using a Make \& Model electrospinning equipment (SprayBase ${ }^{\circledR}$, Ireland). Polymers were made up at specific concentrations and then mixed prior to electrospinning. PVA was prepared at a concentration of $9 \% \mathrm{w} / \mathrm{v}$ in $\mathrm{ddH}_{2} \mathrm{O}, \mathrm{ALG}$ was prepared at $2 \% \mathrm{w} / \mathrm{v}$ in $\mathrm{ddH}_{2} \mathrm{O}$, and $\mathrm{CHT}$ was prepared at $2 \% \mathrm{w} / \mathrm{v}$ in a $1 \%$ acetic acid solution. Both PVA-ALG and PVA-CHT solutions was prepared at a ratio of 9:1 and mixed continually for $24 \mathrm{~h}$ prior to electrospinning. Parameters utilised for the electrospinning of ALG-PVA and CHT-PVA patches included an applied voltage of $8 \mathrm{kV}$, a flow rate of $4 \mu \mathrm{L} / \mathrm{min}$, and a working height of $8 \mathrm{~cm}$. The syringe containing the ALG-PVA solution was subjected to these conditions for $1 \mathrm{~h}$ prior to a change over, where the syringe containing the CHT-PVA solution was electrospun for a further $1 \mathrm{~h}$, which resulted in the fabrication of a bilayer ALG/PVACHT/PVA wound patch.

\section{Crosslinking of Bilayered Electrospun Nanofibre Wound Patch}

Crosslinking was conducted in order to confer stability of the bilayer wound patch in aqueous conditions, allowing the controlled release of nanoparticles. A crosslinking solution of glutaraldehyde (GA) (Sigma Aldrich, UK) in methanol (Sigma Aldrich, UK) was prepared at a concentration of $1 \%$. Following electrospinning, the bilayered wound patch was soaked in 
the crosslinking solution for $24 \mathrm{~h}$. Thereafter, each bilayered wound patch was washed with methanol (three times) and then washed with ultra-pure water (three times). Finally, each bilayer wound patch was dried in air under ambient conditions for $24 \mathrm{~h}$. The morphology of the bilayered wound patch was examined using a Scanning Electron Microscopy (Philips, USA) operated at an acceleration voltage of $15 \mathrm{kV}$.

Fourier transform infrared (FTIR) analysis of Bilayered Electrospun Nanofibre Wound Patch

Fourier transform infrared (FTIR) spectroscopy (Nicolet 1S10 FT-IR spectrometer, Thermo Fisher, USA) was used to identify the presence of specific chemical groups (i.e. PVA, ALG and $\mathrm{CHT}$ ) within the bilayered wound patch and to determine the extent of crosslinking. Samples were analysed over 120 scans between 500 and $4000 \mathrm{~cm}^{-1}$ and averaged at $4 \mathrm{~cm}^{-1}$ resolution.

\section{Direct Polymer-associated Toxicity effect on Cell Proliferation with Bilayered Electrospun Nanofibre Wound Patch}

The direct cellular response was also evaluated to analyse if the polymer had an effect on the proliferation rate of cells. Briefly, HMEC-1 endothelial cells were seeded in 96-well plates at a density of $10 \times 10^{3}$ cells per well containing the crosslinked bilayered wound patch or untreated control $(1 \mathrm{~cm} \times 1 \mathrm{~cm})$. The cells were incubated with $200 \mu \mathrm{L}$ of growth medium at $37^{\circ} \mathrm{C}$ for $24 \mathrm{~h}$. The medium was then removed, and cells fixed for $10 \mathrm{~min}$ in a $4 \%$ formaldehyde solution (Sigma Aldrich, UK). DAPI reagent (Thermo Fisher, USA) was then added to each well and incubated for $30 \mathrm{~min}$. Each well was subsequently imaged and cell population numbers counted within an area of $1 \mathrm{~mm} \times 1 \mathrm{~mm}$ and normalised to the untreated control group. 


\section{Indirect Polymer-associated Toxicity assay in vitro with Bilayered Electrospun Nanofibre}

\section{Wound Patch}

The biocompatibility of the bilayer wound patch is an important parameter to ensure no compromise is made to the already delicate wound tissue. The indirect cytotoxic response of the crosslinked bilayered wound patch was assessed using the MTS assay. Specifically, each wound patch $(1 \mathrm{~cm}$ by $1 \mathrm{~cm})$ was incubated in Opti-MEM medium (Thermo Fisher, USA) at $37^{\circ} \mathrm{C}$ for $48 \mathrm{~h}$. HMEC-1 endothelial cells were then seeded in $96-$ well plates at a density of 10 $\mathrm{x} 10^{3}$ cells per well. Following the incubation period, the culture medium was replaced with the prepared extraction medium and incubated for an additional $24 \mathrm{~h}$. The MTS reagent (Sigma Aldrich, UK) was then added to each well and incubated for $2 \mathrm{~h}$. Absorbance was subsequently recorded at $490 \mathrm{~nm}$ using an OmniStar plate reader (BioTek Instruments Inc., UK). Cell viability is presented as a percentage relative to untreated control.

\section{Nanoparticle Release from Bilayered Electrospun Nanofibre Wound Patch}

The release of the RALA/siFKBPL nanoparticles from the bilayered electrospun nanofibre wound patch is an important parameter to evaluate the concentration of therapeutic released over time. The crosslinked bilayered electrospun nanofibre wound patch was cut into $1 \mathrm{x} 1 \mathrm{~cm}$ sections. Thereafter, RALA/siFKBPL nanoparticles were synthesised as described previously and pipetted onto wound patch in a soak and dry approach over a $24 \mathrm{~h}$ period. The Quanti-iT Picogreen assay (Life Technologies, UK) was used to determine the extent of RALA/siFKBPL nanoparticles released from the crosslinked bilayered wound patch. Each wound patch containing $10 \mu \mathrm{g}$ of RALA/siFKBPL nanoparticles was placed in $5 \mathrm{~mL}$ ultrapure water (Thermo Fisher, USA) and samples were extracted at predefined time points up to $48 \mathrm{~h}$. 
Samples were then incubated with Proteinase-K (Thermo Fisher, UK) for 90 min at $37^{\circ} \mathrm{C}$ to dissociate the nanoparticles and the nanoparticle release was then determined.

\section{Degradation of Bilayered Electrospun Nanofibre Wound Patch}

It is important to evaluate the rate in which the bilayered wound patch degrades as this will have an influence on nanoparticle release. The degradation profile of the crosslinked bilayered wound patch in PBS (pH 7.4) was evaluated over 48 h. At pre-defined intervals, each wound patch was removed from the PBS, washed with fresh PBS, and subsequently dried in an air oven at $40^{\circ} \mathrm{C}$ for $24 \mathrm{~h}$. The degradation index $\left(D_{i}\right)$ was determined using equation 1:

$$
D_{i}=\frac{W_{0}-W_{t}}{W_{0}} * 100
$$

where $W_{0}$ refers to the original weight and $W_{t}$ is the dry weight of each sample after incubation.

\section{In vivo Wound Closure Assay (Murine Model)}

\section{Wounding Surgery}

In vivo application of the RALA/siFKBPL loaded bilayered wound patch was conducted to assess the translation of in vitro findings into complex organisms, an important step in pre-

clinical evaluation. $\mathrm{C} 57 \mathrm{BL} / 6 \mathrm{~J}$ mice were anaesthetised using 5\% Isoflurane prior to hair being removed from the rear dorsum using clippers and cream (Nair, UK). The surgical site was disinfected using $70 \%$ alcohol. Thereafter, the dorsal skin was folded and raised between the index finger and thumb to create a skinfold. The mouse was placed in the lateral position, and a $5 \mathrm{~mm}$ diameter sterile disposable biopsy punches (Stiefel, UK) was used to remove two skin layers completely. This created full-thickness symmetrical wounds. The day of surgery was 
considered Day 0 of the study. Following surgery, the wounds were treated with different groups of the bilayered wound patch (i.e. ALG-CHT-PVA, ALG-CHT-PVA loaded with RALA/siFKBPL nanoparticles, ALG-CHT-PVA loaded with siFKBPL, and a control dressing (Algisite ${ }^{\circledR}$, Smith \& Nephew, UK)). The latter wound dressing is composed of calcium alginate and is a fibrous dressing and that forms a gel under aqueous conditions. Following placement of the bilayered wound patch, a secondary dressing of a commercial transparent film Tegaderm $^{\mathrm{TM}}(3 \mathrm{M}$, Ireland) was placed over each wound to secure the patch in position. Postsurgery, animals were housed individually, moved to a warm area for recovery from anaesthesia, and monitored for $24 \mathrm{~h}$. Once fully recovered, the animals were moved to routine housing.

\section{Histological Analysis}

Following euthanasia (Day 7), each wound bed was harvested and fixed in 10\% formalin for $24 \mathrm{~h}$ prior to pathologic examination. Each tissue section was embedded in paraffin and cut into $3 \mu \mathrm{m}$ sections that were placed onto a glass microscope slides (Northern Ireland BioBank). The samples were processed for hematoxylin and eosin (H\&E) staining. The number of blood vessels and vessel diameter was quantified from randomly selected areas of tissue samples ImageJ (version 1.52a, NIH, USA).

\section{Statistical analysis}

Unless otherwise stated, three independent experiments were conducted for analysis of data from the nanoparticle/nanofibre characterisation and the in vitro studies. The results are presented as mean $\pm \operatorname{SEM}(\mathrm{n}=3)$. For in vivo experimentation, five-independent experiments were conducted for each analysis and the results are presented as mean $\pm \operatorname{SEM}(n=5)$. Statistically significant differences were computed using either one-way or two-way analysis 
of variance and a $p$-value of $\leq 0.05$ being considered as significant. Statistical posthoc tests for individual experiments are detailed in figure legends. Statistical analysis was performed using Prism 8.0 (GraphPad Software, CA, USA). 


\section{Results}

\section{Characterisation of the RALA/siFKBPL Nanoparticle Complexes}

Particle size and charge are critical factors for cellular uptake with only submicron cationic particles effectively endocytosed [28]. The hydrodynamic size and zeta potential of the particles were therefore assessed by measuring siFKBPL condensation by RALA (Figure 1A). RALA condensed the siFKBPL into nanoparticles $<100 \mathrm{~nm}$ from N:P 1 upwards. The stability of the RALA/siFKBPL (N:P 6) complexes over a temperature range was also investigated (Figure 1B). The complex size remained consistently $<70 \mathrm{~nm}$ when exposed to temperatures ranging from $4-37^{\circ} \mathrm{C}$. These results suggest that RALA condensed the siFKBPL into stable nanoparticles that preserved the ideal characteristics for intracellular uptake with respect to size at $4^{\circ} \mathrm{C}$, room, and body temperature. TEM imaging of RALA/siFKBPL complexes at N:P 6 confirmed the nanoparticle size to be $<100 \mathrm{~nm}$ and to be uniform in shape (Figure 1C). Following the synthesis of RALA/siFKBPL complexes, analysis by gel retardation indicated that the siFKBPL was impeded from migrating down of the agarose gel from N:P 4 onwards (Figure 1D) as the visibility of the siFKBPL in the gel was undetectable. This was indicative of decreased fluorescence due to the RALA peptide preventing EtBr intercalation with the siFKBPL as a consequence of increased complexation.

\section{Transfection of HMEC-1 Endothelial cells with RALA/pEGFP-N1 nanoparticles}

Having established that RALA/siFKBPL complexes exhibited suitable characteristics for efficient cellular uptake, the transfection efficacy was assessed in vitro via fluorescent microscopy and flow cytometric analysis. Using RALA/pEGFP-N1 nanoparticles at a N:P ratio of 6, transfection efficiency was $33.7 \pm 1.65 \%$ in HMEC-1 endothelial cells (Figure 2A), which is visually represented in (Figure 2C). These transfection efficacies were less cytotoxic at lower N:P ratios (Figure 2B). At N:P ratios of 8-12 some toxicity was observed, 
but this could be attributed to an excess of unbound peptide, which was not required. Taken together, these results indicate that complexes at $\mathrm{N}: \mathrm{P} 6$ demonstrated the highest transfection efficiency without any significant compromise to the cell viability, and therefore all future experiments were conducted at an N:P ratio of 6.

FKBPL knock-down using RALA/siFKBPL in HMEC-1 Endothelial Cells and the Subsequent Effects on Cell Migration and Tubule Formation in vitro

It has previously been shown that FKBPL is secreted from HMEC-1 endothelial cells (18). To demonstrate effective knockdown of siFKBPL, western blot analysis was conducted using HMEC-1 cells transfected with either RALA/siSC or RALA/siFKBPL (Figure 2D). Results indicated there was significant knockdown compared to untreated controls at $48 \mathrm{~h}$ and that RALA did not impact FKBPL as evidenced in the scrambled controls $(*, p<0.05)$. To complement this, an in vitro wound scratch assay was performed to evaluate endothelial cell migration (Figure 3). Significant acceleration of wound closure was demonstrated when the HMEC-1 cells were treated with RALA/siFKBPL at $8 \mathrm{~h}\left({ }^{* * *}, \mathrm{p}<0.001\right)$ where an average percentage closure of $69.7 \pm 18.8 \%$ was observed, compared to untreated which had closed $35.8 \pm 6.47 \%$. At $24 \mathrm{~h}$ a significant closure $(* * * *, \mathrm{p}<0.0001)$ of $100 \%$ was also observed in the RALA/siFKBPL group when compared to the untreated control group which showed closure of $32.5 \pm 10.96 \%$. The tubule formation assay was employed to assess the impact of RALA/siFKBPL nanoparticles on angiogenesis (Figure 4). Tubule number was significantly higher for the RALA/siFKBPL nanoparticle group compared to untreated control and RALA/siSC groups at $8 \mathrm{~h}(*, \mathrm{p}<0.05)$ and $24 \mathrm{~h}\left({ }^{* * *}, \mathrm{p}<0.001\right)$ after plating. 


\section{Bilayered Electrospun Nanofibre Wound Patch Fabrication}

The bilayered electrospun nanofibres were fabricated as indicated in Figure 5A, and subsequently crosslinked using GA in methanol (Figure 5B). The SEM images for the bilayered wound patch pre- and post-crosslinking treatment are shown in Figure 5C. It was observed that the wound patch maintained a fibrous structure after crosslinking with GA/methanol. FTIR spectroscopy of the crosslinked bilayered wound patch confirmed the presence of alginate and chitosan, which was indicated by the $\mathrm{C}=0$ stretch and $\mathrm{CH}_{3}$ symmetric deformation bonds at $1732 \mathrm{~cm}^{-1}$ and $1373 \mathrm{~cm}^{-1}$ (Figure 5D). Additionally, effective crosslinking reaction was confirmed via the formation of acetal ring between the hydroxyl groups of the PVA and aldehyde groups of the GA as at 2850 and $2750 \mathrm{~cm}^{-1}$ as $\mathrm{C}-\mathrm{H}$ stretching is evident, and is related to aldehydes, a duplet absorption with peaks attributed to the alkyl chain.

Direct Polymer Associated effect on cell proliferation and indirect Polymer Associated Toxicity in vitro with the Bilayered Electrospun Nanofibre Wound Patch

The polymer associated toxicity was tested in vitro with HMEC-1 cells directly by seeding onto the nanofibres and indirectly by exposing cells to media in which the patch had been incubated in for $48 \mathrm{~h}$ and the effect on proliferation was assessed by directly seeding cells onto the nanofibres (Figure 5E-5G). No toxicity biocompatibility issues were observed with results comparable to untreated cells reported in both scenarios. This is an highly positive result as the biocompatibility of the patch could be rate-limiting, as it is applied to preexisting compromised tissue [30]. 


\section{Release of RALA/siFKBPL complexes from the Bilayered Electrospun Nanofibre Wound Patch}

RALA/siFKBPL nanoparticle release was determined using a standard curve (of known siFKBPL concentrations) within $48 \mathrm{~h} .100 \%$ of the siFKBPL was recovered within this period (Figure 6A). Degradation was determined by calculating the polymer mass loss with $48 \mathrm{~h}$ (Figure 6B). It was evident that minimal mass loss occurred within the initial $48 \mathrm{~h}$, with $>90 \%$ of the polymer remaining. The relative slow degradation rate can be explained by the positive influence of the crosslinking process. Taking these results together indicated the RALA/siFKBPL nanoparticles are being released via swelling as opposed to polymer degradation.

In vivo evaluation of Bilayered Electrospun Nanofibre Wound Patch mediated delivery of RALA/siFKBPL

All wounds, irrespective of treatment, were essentially fully contracted by Day 7 post-surgery. A schematic representation of the protocol is detailed in Figure 7A, and a representative histology section of mouse skin with arterial and venous structures identified as an angiogenic measures (Figure 7B). As shown in Figure 7C(i-ii) the vessel density increased significantly for wounds treated with the bilayered wound patch loaded with RALA/siFKBPL nanoparticles $(* * *, p<0.001)$ compared to all other treatments including the commercial control (Algisite $\left.{ }^{\circledR}\right)$. With respect to vessel diameter, a non-significant increase was observed for the bilayered wound patch loaded with RALA/siFKBPL nanoparticles group compared to the untreated group. Histological imaging of the epidermal layer of the wound provided visual support of an increase in blood vessel density for the bilayered wound patch loaded with RALA/siFKBPL nanoparticles compared to the untreated group (Figure 7D(i-ii)). 


\section{Discussion}

The work outlined in this paper shows for the first time that RALA can effectively delivery siFKBPL for wound healing applications. The delivery of siFKBPL using RALA exhibited significant increases in in vitro functionality of HMEC-1 cells, via the tubule formation and wound scratch assay. Incorporation of these nanocomplexes into the bilayered ALG-CHTPVA wound patches was found to be a promising platform for therapeutic delivery to fullthickness wounds of C57BL/6J mice in vivo.

The condensation of siFKBPL by RALA complexation occurred from a N:P ratio of 1 . This confirms previous studies which characterised RALA complexed with a range of nucleic acid cargoes [8-9]. Hydrodynamic size analysis coupled with TEM revealed that the nanoparticles from N:P 6 onwards exhibited biophysical characteristics within the ideal size and charge range for receptor-mediated endocytosis [30-31]. Additionally, there was no significant change in particle size over a range of temperatures, further showing the highly stable nature of the RALA/ siFKBPL nanoparticles. For the efficient delivery of genetic cargo it is important that these parameters are met, otherwise the transfection efficiency will be compromised, or there may be toxicity implications [32-33]. For example, it is well noted that positively charged nanoparticle results in higher rates of cell internalisation compared to neutral and negatively charged counterparts. Indeed, Huhn et al. conducted charge-dependent interaction studies with colloidal gold nanoparticles in human umbilical vein endothelial cells. The authors found that when cationic, the nanoparticle internalisation was higher compared to anionic counterparts [35]. 
The intracellular delivery of therapeutics can significantly heighten potency. RALA is $\mathrm{pH}-$ responsive, which enables cargo release by degradation in endosomes post-endocytosis and excellent cytoplasmic delivery. In this study, we have shown that charge neutralisation and condensation of siFKBPL can facilitate its transport across the cell membrane of endothelial cells. Transfection of HMEC-1 cells showed that RALA mediated delivery of nucleic acids without impacting cell viability at N:P ratio of 6 . Lack of toxicity is an important parameter in non-viral gene delivery, particularly for wound healing as damage to the already delicate wound tissue would be rate-limiting. Cationic nanoparticles result in enhanced cellular internalisation; however, a positive charge can also cause toxicity problems. With this in mind, it was important to conduct cytotoxicity studies in parallel with transfection studies in order to determine the optimal N:P ratio (in this case $\mathrm{N}: \mathrm{P}$ 6) for transfection without compromise to viability.

During the normal wound healing process, endothelial cells migrate through the granulation tissue to partake in angiogenesis, a process that is paramount for efficient healing [36]. In a chronic wound setting these cells can often be senescent, leading to a lack of angiogenesis and hence hypoxia [37]. The delivery of siFKBPL in HMEC-1 cells resulted in a significant decrease in the endogenous protein levels of FKBPL, which correlated in a significant increase in cellular function as evidenced by an upsurge in cell migration and tubule forming capacity, this outcome is reliant on the function of siFKBPL to enhance the levels of the cell surface receptor CD44 [9]. The main issues with restorative endothelial cells in a chronic wound are the lack of migration and subsequent angiogenesis and these have both been negated in this study [38]. The in vitro functionality results presented in this study are promising, but it would be necessary to further explore the downstream molecular mechanism of FKBPL silencing in other relevant skin cells. 
The crosslinking of the bilayered wound patch did not compromise the wound microenvironment or affect HMEC-1 cellular viability and proved an effective vehicle for controlled therapeutic cargo release. Other studies have reported similar findings for PVAbased drug delivery systems. Alhosseini et al. successfully demonstrated the proliferation of PC12 nerve cells on PVA nanofibrous scaffolds [39]. Additionally, Cole et al. designed PVAbased microneedles for transdermal delivery of RALA/DNA nanoparticles and reported no issues with the viability of NCTC-929 fibroblast cells at concentrations up to $40 \mathrm{mg} / \mathrm{mL}$ [40]. Although the polymers used for this study all have noted biocompatibility, it would be prudent in future work to determine whether or not they would be replaced by tissue in vivo or evoke a possible foreign body response if applied long term. If such complications were to arise, it could lead to difficulties in healing.

Although scaffold-based non-viral gene delivery offers many potential applications, only a few examples exist for incorporating nucleic acids within electrospun scaffolds. Luu et al., for example, investigated the loading of naked pDNA into electrospun nanofibres composed of (PLGA) random copolymer and a poly(d-1-lactide)-poly (ethylene glycol) (PLA-PEG) block copolymer with a loading efficiency of between $68-80 \%$ of the cargo. The pDNA was found to be released over a 20 -day period with an initial burst release of up to $36 \%$ within the first 15 min. This study also concluded that the maximum noted transfection efficiency from the nanofibres was approximately 4\% in preosteoblast murine cells (MC3T3) [41]. Utilising RALA to protect the siFKBPL from degradation, in addition to ensuring effective intracellular delivery, resulted in a loading efficiency of $100 \%$, with complete release of the nanoparticles over a $48 \mathrm{~h}$ period. In a study conducted by Monteforte et al., glypican-1 was identified to have a reduced level in skin blood vessels in patients with type 2 diabetes. Consequently Monteforte 
et al. developed a glypican-1 protein therapy, delivered using a lipofectamine 2000 in an alginate hydrogel. When the glypican-1 complexes were delivered in combination with fibroblast growth factor-2 in vitro into Human umbilical vein endothelial cells (HUVECs), it resulted in an increase in branch points, tubule length, and number of tubules formed $(p<0.05)$ [42]. These results are positive with an enhancement in angiogenesis; however, use of lipofectamine 2000 can result in toxicity to cells, and in a delicate wound environment this would be counterproductive [42-43]. Indeed, in our study, we observed that the use of lipofectamine had significant effects on HMEC-1 viability. Comparatively, treatment with RALA/siFKBPL nanoparticles resulted in a more pronounced enhancement in the tubular forming capacity in vitro compared to those stated by the authors. The use of RALA has negligible effects on cell viability at a $\mathrm{N}: \mathrm{P}$ ratio of 6 , making it a more appropriate transfection agent for wound healing applications and explains the highly significant angiogenic response. This has been substantiated by Kobsa et al., who developed an electrospun construct manufactured from a blend of PLA or PCL. These nanofibres were soaked in PEI solution and loaded with plasmids which encode for keratinocyte growth factor (KGF), also by soaking. These constructs were tested in vivo via the full thickness wound model in C57BL/6J mice [45]. The authors found that compared to scaffolds containing control plasmid, the pKGF loaded nanofibres resulted in a significant enhancement in the rate of re-epithelialisation and keratinocyte proliferation. The resultant effect on the epidermal layer was a $110 \%$ increase in the average thickness. There were no noted differences in epidermal thickness between untreated wounds and wounds treated with constructs containing no pKGF. Although these are very positive results, keratinocytes are the only cell type here to benefit from such a treatment. The delivery of siFKBPL using the RALA peptide offers as a superior solution as it stimulates angiogenesis, a process from which all cell types in the wound can benefit due to enhanced oxygen and nutrient delivery in situ. PEI of a molecular weight of $25 \mathrm{kDa}$ is considered by 
many as the gold standard of gene delivery [45-46]. However, in a similar way to the use of lipofectamine, PEI has noted toxicity in vitro and in vivo, making it clinically redundant for wound healing [47-49]. This further supports the use of the RALA peptide, as a non-cytotoxic superior transfection agent to that of commercially available counterparts.

The in vivo murine wound model demonstrated that the delivery of the RALA/siFKBPL nanoparticles from the bilayered wound patch offered significant pro-angiogenic effects with an increase in vessel number of $325.8 \%$ compared to the untreated controla. Alginates are known to maintain moisture within wounds, and thus help promote healing through, for example, facilitating autolytic debridement, reducing the possibility of infection and preservation of growth factors within the wound fluid [50-52]. When wounds were treated with the control dressing (Algisite ${ }^{\circledR}$ ), the angiogenic response was similar to that of wound treated with the unloaded bilayered ALG-CHT-PVA wound patch, as well as the untreated control. This is a highly encouraging result, as it signifies that the nanofibres developed through this research are comparable to commercial equivalents and did not hinder angiogenesis.

The use of the bilayer wound patch to deliver RALA/siFKBPL nanoparticles in vivo mirrored the in vitro findings and is a promising for the future development of this nanomedicine for the treatment of wounds. Other research has explored the topical of administration of therapeutic proangiogenics such as VEGF. Galiano et al., for example, treated diabetic $\mathrm{db} / \mathrm{db}$ mice with a topical VEGF, resulting in complete resurfacing of the wounds by Day 12 post-surgery compared to controls of phosphate-buffered saline (PBS) and no treatment. This was a comparable result to that rate of wound resurfacing in nondiabetic mice $\sim$ Day 10 . However, for this effect to be seen, a daily application containing $20 \mu \mathrm{g}$ of recombinant human VEGF165 protein was required [54]. Ergo, large quantities of modified recombinant VEGF 
protein are required, making this an extremely expensive therapy option. Our study showed significant improvement in the angiogenic profile of mice with two applications of RALA/siFKBPL loaded nanofibres containing $20 \mu \mathrm{g}$ of siFKBPL cargo. The advantage of using these nanoparticle-based systems is the enhanced cellular delivery facilitated by the RALA peptide. Thus an effect occurs with lower doses. However, it would be prudent that in future work, a db/db murine model for delayed wound healing was also tested in vivo [54-55]. Furthermore, it would be judicious to test the nanofibre device in a porcine wound model. Pigs are anatomically and physiologically comparable to humans, thus make for excellent models of human diseases [56-57]. Indeed, the skin of humans and pigs are similar in that they have a comparatively thick epidermis and dermal papillae [59]. This model could, therefore, be used to test our device in vivo to measure not only angiogenesis but also reepithelisation and wound closure, outputs of which can be at times, unreliable in murine studies due to differing skin architectures [60], [61].

Our data demonstrates the exciting potential of the RALA/siFKBPL nanoparticle-loaded bilayer wound patches as an angiogenic therapy for wound healing. The results indicate the merits of the medical device in vitro, through enhancement of cell migration as well as tubular forming capacity in HMEC-1 cells. Furthermore, following in vivo testing, it was evident that our nanoparticle-loaded bilayered wound patch significantly enhanced angiogenesis compared to untreated and commercial (Algisite $\left.{ }^{\circledR}\right)$ controls.

\section{Conclusion}

Considerable data indicate that RALA/siFKBPL nanoparticle delivery from bilayered ALG/PVA-CHT/PVA wound patches significantly enhanced angiogenesis in vitro and in vivo with negligible toxicity and proved superior to commercially available and clinically relevant 
controls. This is further evidence to support the utility of the RALA platform as a possible medical device directed towards the treatment of wounds.

\section{References}

[1] E. J. Mulholland, N. Dunne, and H. O. McCarthy, 'MicroRNA as Therapeutic Targets for Chronic Wound Healing', Mol. Ther. - Nucleic Acids, vol. 8, pp. 46-55, Sep. 2017.

[2] S. Guo and L. A. Dipietro, 'Factors affecting wound healing', J. Dent. Res., vol. 89, no. 3, pp. 219-229, Mar. 2010.

[3] G. S. Schultz, G. A. Chin, L. Moldawer, and R. F. Diegelmann, Principles of Wound Healing. 2011.

[4] P. Bao, A. Kodra, M. Tomic-Canic, M. S. Golinko, H. P. Ehrlich, and H. Brem, 'The Role of Vascular Endothelial Growth Factor in Wound Healing', J. Surg. Res., vol. 153, no. 2, pp. 347-358, May 2009.

[5] W. He, Z. Ma, T. Yong, W. E. Teo, and S. Ramakrishna, 'Fabrication of collagencoated biodegradable polymer nanofiber mesh and its potential for endothelial cells growth', Biomaterials, vol. 26, no. 36, pp. 7606-7615, 2005.

[6] R. J. Ruthenborg, J.-J. Ban, A. Wazir, N. Takeda, and J.-W. Kim, 'Regulation of wound healing and fibrosis by hypoxia and hypoxia-inducible factor-1.', Mol. Cells, vol. 37, no. 9, pp. 637-43, Sep. 2014.

[7] A. Yakkundi et al., 'FKBPL is a critical antiangiogenic regulator of developmental and pathological angiogenesis.', Arterioscler. Thromb. Vasc. Biol., vol. 35, no. 4, pp. 84554, Apr. 2015.

[8] H. O. McCarthy et al., 'Development and characterization of self-assembling 
nanoparticles using a bio-inspired amphipathic peptide for gene delivery.', J. Control. Release, vol. 189, pp. 141-9, Sep. 2014.

[9] R. Bennett et al., 'RALA-mediated delivery of FKBPL nucleic acid therapeutics', Nanomedicine, vol. 10, no. 19, pp. 2989-3001, Oct. 2015.

[10] C. M. McCrudden et al., 'Gene therapy with RALA/iNOS composite nanoparticles significantly enhances survival in a model of metastatic prostate cancer', Cancer Nanotechnol., vol. 9, no. 1, p. 5, Dec. 2018.

[11] A. A. Ali et al., 'DNA vaccination for cervical cancer; a novel technology platform of RALA mediated gene delivery via polymeric microneedles', Nanomedicine Nanotechnology, Biol. Med., vol. 13, no. 3, pp. 921-932, Apr. 2017.

[12] A. S. Massey et al., 'Potentiating the Anticancer Properties of Bisphosphonates by Nanocomplexation with the Cationic Amphipathic Peptide, RALA.', Mol. Pharm., vol. 13, no. 4, pp. 1217-28, Apr. 2016.

[13] H. Moghadas, M. S. Saidi, N. Kashaninejad, A. Kiyoumarsioskouei, and N.-T. Nguyen, 'Fabrication and characterization of low-cost, bead-free, durable and hydrophobic electrospun membrane for 3D cell culture.', Biomed. Microdevices, vol. 19, no. 4, p. 74, Aug. 2017.

[14] P. Bhattarai, K. B. Thapa, R. B. Basnet, and S. Sharma, 'Electrospinning: How to Produce Nanofibers Using Most Inexpensive Technique? An Insight into the Real Challenges of Electrospinning Such Nanofibers and Its Application Areas', Int. J. Biomed. Adv. Res., vol. 5, no. 9, p. 401, Sep. 2014.

[15] M. Gizaw, J. Thompson, A. Faglie, S.-Y. Lee, P. Neuenschwander, and S.-F. Chou, 'Electrospun Fibers as a Dressing Material for Drug and Biological Agent Delivery in Wound Healing Applications', Bioengineering, vol. 5, no. 1, p. 9, Jan. 2018.

[16] A. M. Al-Enizi, M. M. Zagho, and A. A. Elzatahry, 'Polymer-Based Electrospun 
Nanofibers for Biomedical Applications.', Nanomater. (Basel, Switzerland), vol. 8, no. 4, Apr. 2018.

[17] R. Bhattarai, R. Bachu, S. Boddu, and S. Bhaduri, 'Biomedical Applications of Electrospun Nanofibers: Drug and Nanoparticle Delivery', Pharmaceutics, vol. 11, no. 1, p. 5, Dec. 2018.

[18] M. Kita, Y. Ogura, Y. Honda, S. H. Hyon, W. Cha, and Y. Ikada, 'Evaluation of polyvinyl alcohol hydrogel as a soft contact lens material.', Graefes Arch. Clin. Exp. Ophthalmol., vol. 228, no. 6, pp. 533-7, 1990.

[19] T. H. Young, N. K. Yao, R. F. Chang, and L. W. Chen, 'Evaluation of asymmetric poly(vinyl alcohol) membranes for use in artificial islets.', Biomaterials, vol. 17, no. 22, pp. 2139-45, Nov. 1996.

[20] S. Maruoka et al., 'Biocompatibility of polyvinylalcohol gel as a vitreous substitute.', Curr. Eye Res., vol. 31, no. 7-8, pp. 599-606.

[21] R. K. Tubbs, 'Sequence distribution of partially hydrolyzed poly(vinyl acetate)', $J$. Polym. Sci. Part A-1 Polym. Chem., vol. 4, no. 3, pp. 623-629, Mar. 1966.

[22] M. M. Gepp et al., 'Bioactive surfaces from seaweed-derived alginates for the cultivation of human stem cells', J. Appl. Phycol., vol. 29, no. 5, pp. 2451-2461, Oct. 2017.

[23] M. J. Chalanqui et al., 'Influence of alginate backbone on efficacy of thermoresponsive alginate-g-P(NIPAAm) hydrogel as a vehicle for sustained and controlled gene delivery', Mater. Sci. Eng. C, vol. 95, pp. 409-421, Feb. 2019.

[24] B. A. Aderibigbe and B. Buyana, 'Alginate in Wound Dressings.', Pharmaceutics, vol. 10, no. 2, Apr. 2018.

[25] I. Younes and M. Rinaudo, 'Chitin and chitosan preparation from marine sources. Structure, properties and applications.', Mar. Drugs, vol. 13, no. 3, pp. 1133-74, Mar. 
2015.

[26] X. ZHU, Q. WANG, J. CAO, and W. JIANG, 'EFFECTS OF CHITOSAN COATING ON POSTHARVEST QUALITY OF MANGO ( MANGIFERA INDICA L. CV. TAINONG) FRUITS', J. Food Process. Preserv., vol. 32, no. 5, pp. 770-784, Oct. 2008.

[27] S. Dhivya, V. V. Padma, and E. Santhini, 'Wound dressings - a review', BioMedicine, vol. 5 , no. 4 , p. 22 , Dec. 2015 .

[28] S. Behzadi et al., 'Cellular uptake of nanoparticles: journey inside the cell', Chem. Soc. Rev., vol. 46, no. 14, pp. 4218-4244, 2017.

[29] A. Valentine et al., 'FKBPL and Peptide Derivatives: Novel Biological Agents That Inhibit Angiogenesis by a CD44-Dependent Mechanism', Clin. Cancer Res., vol. 17, no. 5, pp. 1044-1056, Mar. 2011.

[30] E. A. Kamoun, E.-R. S. Kenawy, and X. Chen, 'A review on polymeric hydrogel membranes for wound dressing applications: PVA-based hydrogel dressings', J. Adv. Res., vol. 8, no. 3, pp. 217-233, May 2017.

[31] N. Yang, 'Nonviral gene delivery system', Int. J. Pharm. Investig., vol. 2, no. 3, p. 97, 2012.

[32] M. Ansar, D. Serrano, I. Papademetriou, T. K. Bhowmick, and S. Muro, 'Biological Functionalization of Drug Delivery Carriers To Bypass Size Restrictions of ReceptorMediated Endocytosis Independently from Receptor Targeting', ACS Nano, vol. 7, no. 12, pp. 10597-10611, Dec. 2013.

[33] N. Voigt, P. Henrich-Noack, S. Kockentiedt, W. Hintz, J. Tomas, and B. A. Sabel, 'Toxicity of polymeric nanoparticles in vivo and in vitro.', J. Nanopart. Res., vol. 16, no. 6, Jun. 2014.

[34] J. Panyam and V. Labhasetwar, 'Biodegradable nanoparticles for drug and gene 
delivery to cells and tissue', Adv. Drug Deliv. Rev., vol. 55, no. 3, pp. 329-347, Feb. 2003.

[35] D. Hühn et al., 'Polymer-Coated Nanoparticles Interacting with Proteins and Cells: Focusing on the Sign of the Net Charge', ACS Nano, vol. 7, no. 4, pp. 3253-3263, Apr. 2013.

[36] M. G. Tonnesen, X. Feng, and R. A. F. Clark, 'Angiogenesis in wound healing.', J. Investig. dermatology. Symp. Proc., vol. 5, no. 1, pp. 40-6, Dec. 2000.

[37] R. G. Frykberg and J. Banks, 'Challenges in the Treatment of Chronic Wounds', Adv. Wound Care, vol. 4, no. 9, pp. 560-582, 2015.

[38] S. Dangwal et al., 'Impairment of Wound Healing in Patients With Type 2 Diabetes Mellitus Influences Circulating MicroRNA Patterns via Inflammatory Cytokines', Arterioscler. Thromb. Vasc. Biol., vol. 35, no. 6, pp. 1480-1488, Jun. 2015.

[39] S. Naghavi Alhosseini, F. Moztarzadeh, S. Kargozar, M. Dodel, and M. Tahriri, 'Development of Polyvinyl Alcohol Fibrous Biodegradable Scaffolds for Nerve Tissue Engineering Applications: In Vitro Study', Int. J. Polym. Mater. Polym. Biomater., vol. 64, no. 9, pp. 474-480, Sep. 2015.

[40] G. Cole et al., 'Dissolving microneedles for DNA vaccination: Improving functionality via polymer characterization and RALA complexation', Hum. Vaccin. Immunother., vol. 13, no. 1, pp. 50-62, Jan. 2017.

[41] Y. K. Luu, K. Kim, B. S. Hsiao, B. Chu, and M. Hadjiargyrou, 'Development of a nanostructured DNA delivery scaffold via electrospinning of PLGA and PLA-PEG block copolymers', J. Control. Release, vol. 89, no. 2, pp. 341-353, Apr. 2003.

[42] A. J. Monteforte et al., 'Glypican-1 nanoliposomes for potentiating growth factor activity in therapeutic angiogenesis.', Biomaterials, vol. 94, pp. 45-56, 2016.

[43] M. A. Hunt, M. J. Currie, B. A. Robinson, and G. U. Dachs, 'Optimizing transfection 
of primary human umbilical vein endothelial cells using commercially available chemical transfection reagents.', J. Biomol. Tech., vol. 21, no. 2, pp. 66-72, Jul. 2010.

[44] M. Bauer et al., 'Toxic effects of lipid-mediated gene transfer in ventral mesencephalic explant cultures', Basic Clin. Pharmacol. Toxicol., vol. 98, no. 4, pp. 395-400, 2006.

[45] S. Kobsa, N. J. Kristofik, A. J. Sawyer, A. L. M. Bothwell, T. R. Kyriakides, and W. M. Saltzman, 'An electrospun scaffold integrating nucleic acid delivery for treatment of full-thickness wounds', Biomaterials, vol. 34, no. 15, pp. 3891-3901, May 2013.

[46] S. Patnaik and K. C. Gupta, 'Novel polyethylenimine-derived nanoparticles for in vivo gene delivery.', Expert Opin. Drug Deliv., vol. 10, no. 2, pp. 215-28, Feb. 2013.

[47] A. Swami, R. K. Kurupati, A. Pathak, Y. Singh, P. Kumar, and K. C. Gupta, 'A unique and highly efficient non-viral DNA/siRNA delivery system based on PEI-bisepoxide nanoparticles.', Biochem. Biophys. Res. Commun., vol. 362, no. 4, pp. 835-41, Nov. 2007.

[48] V. Kafil and Y. Omidi, 'Cytotoxic impacts of linear and branched polyethylenimine nanostructures in a431 cells.', Bioimpacts, vol. 1, no. 1, pp. 23-30, 2011.

[49] B. I. Florea, C. Meaney, H. E. Junginger, and G. Borchard, 'Transfection efficiency and toxicity of polyethylenimine in differentiated Calu-3 and nondifferentiated COS-1 cell cultures.', AAPS PharmSci, vol. 4, no. 3, p. E12, 2002.

[50] M. Breunig, U. Lungwitz, R. Liebl, and A. Goepferich, 'Breaking up the correlation between efficacy and toxicity for nonviral gene delivery', Proc. Natl. Acad. Sci., vol. 104, no. 36, pp. 14454-14459, Sep. 2007.

[51] [Online]. Available: https://www.woundsource.com/blog/benefits-moist-woundhealing. [Accessed: 28-May-2019].

[52] A. Agarwal, J. F. McAnulty, M. J. Schurr, C. J. Murphy, and N. L. Abbott, 'Polymeric materials for chronic wound and burn dressings', in Advanced Wound Repair 
Therapies, Elsevier, 2011, pp. 186-208.

[53] M. Ip, 'Antimicrobial dressings', in Advanced Wound Repair Therapies, Elsevier, 2011, pp. 416-449.

[54] R. D. Galiano et al., 'Topical vascular endothelial growth factor accelerates diabetic wound healing through increased angiogenesis and by mobilizing and recruiting bone marrow-derived cells.', Am. J. Pathol., vol. 164, no. 6, pp. 1935-47, Jun. 2004.

[55] J. Michaels et al., ' $\mathrm{db} / \mathrm{db}$ mice exhibit severe wound-healing impairments compared with other murine diabetic strains in a silicone-splinted excisional wound model.', Wound Repair Regen., vol. 15, no. 5, pp. 665-70.

[56] G. Zhao et al., 'Delayed wound healing in diabetic $(\mathrm{db} / \mathrm{db})$ mice with Pseudomonas aeruginosa biofilm challenge: a model for the study of chronic wounds.', Wound Repair Regen., vol. 18, no. 5, pp. 467-77.

[57] T. P. Sullivan, W. H. Eaglstein, S. C. Davis, and P. Mertz, 'The pig as a model for human wound healing.', Wound Repair Regen., vol. 9, no. 2, pp. 66-76.

[58] M. Fricker, A. Deane, and P. M. Hansbro, 'Animal models of chronic obstructive pulmonary disease.', Expert Opin. Drug Discov., vol. 9, no. 6, pp. 629-45, Jun. 2014.

[59] W. MONTAGNA and J. S. YUN, 'THE SKIN OF THE DOMESTIC PIG.', J. Invest. Dermatol., vol. 42, pp. 11-21, Jul. 1964.

[60] M. S. Hu et al., 'An Improved Humanized Mouse Model for Excisional Wound Healing Using Double Transgenic Mice', Adv. Wound Care, vol. 7, no. 1, pp. 11-17, Jan. 2018

[61] X. Wang, J. Ge, E. E. Tredget, and Y. Wu, 'The mouse excisional wound splinting model, including applications for stem cell transplantation', Nat. Protoc., vol. 8, no. 2, pp. 302-309, Feb. 2013. 
A

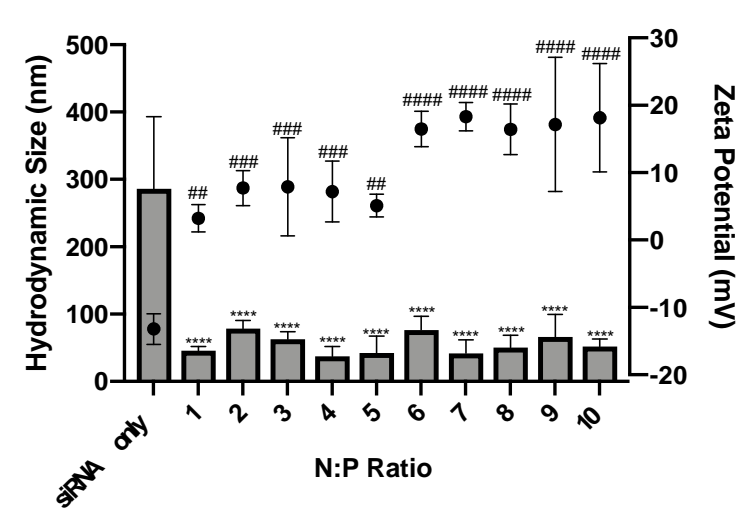

C

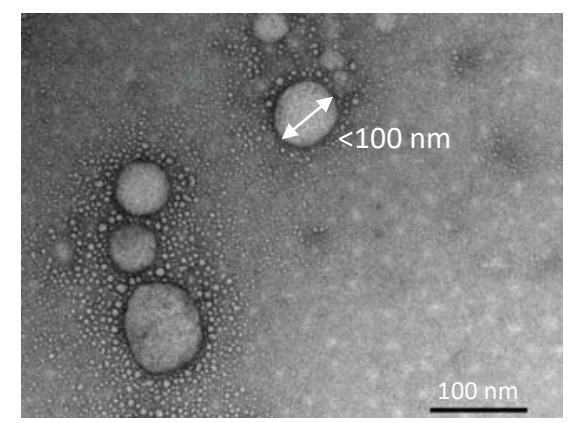

D
B

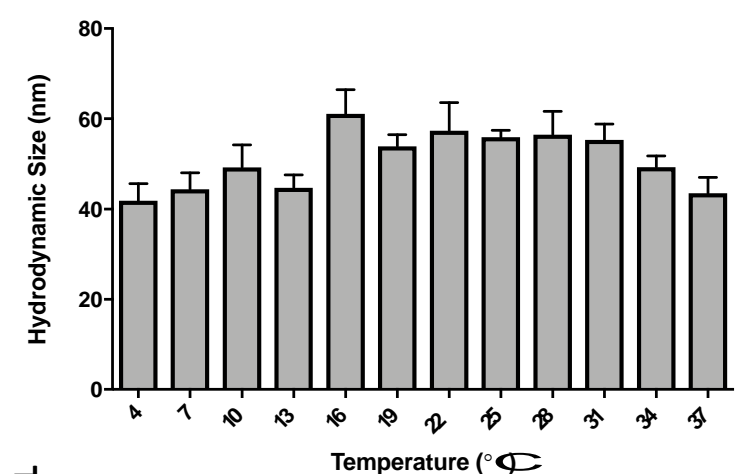

N:P Ratio

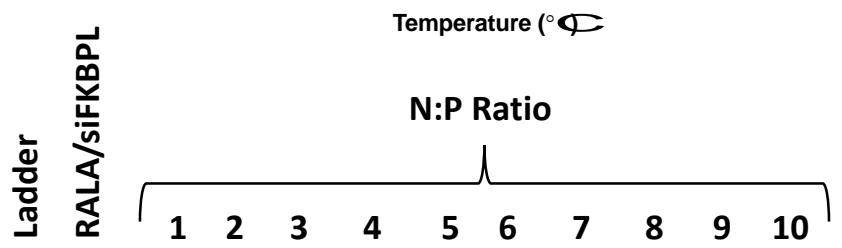

Figure 1: Characterisation of RALA/siFKBPL nanoparticles. (A) Nanoparticles were made up of $50 \mu \mathrm{L}$ with molecular grade water and incubated for $30 \mathrm{~min}$ at room temperature before hydrodynamic size, and zeta potential was measured using a Malvern Zetasizer Nano ZS instrument. Significance is denoted using '*' for Hydrodynamic size and '\#' for Zeta potential; (B) RALA/siFKBPL nanoparticles were synthesised and DLS analysed the stability over a range of temperatures $\left(4-37^{\circ} \mathrm{C}\right)$; (C) TEM image of RALA/siFKBPL complexes N:P ratio 6 (Scale bar $100 \mathrm{~nm}$ ); (D) Gel were prepared using agarose gel $(1 \% \mathrm{w} / \mathrm{v}$ and $0.25 \mu \mathrm{g} / \mathrm{mL}$ ethBr) with electrophoresis showing the mobility of siFKBPL (38 bp) when combined with RALA at different $\mathrm{N}: \mathrm{P}$ ratios 1-10. Results displayed as mean $\pm \mathrm{SEM}, \mathrm{n}=3$ and statistical significance calculated by means of one-way ANOVA with Dunnett post-hoc tests. 
A

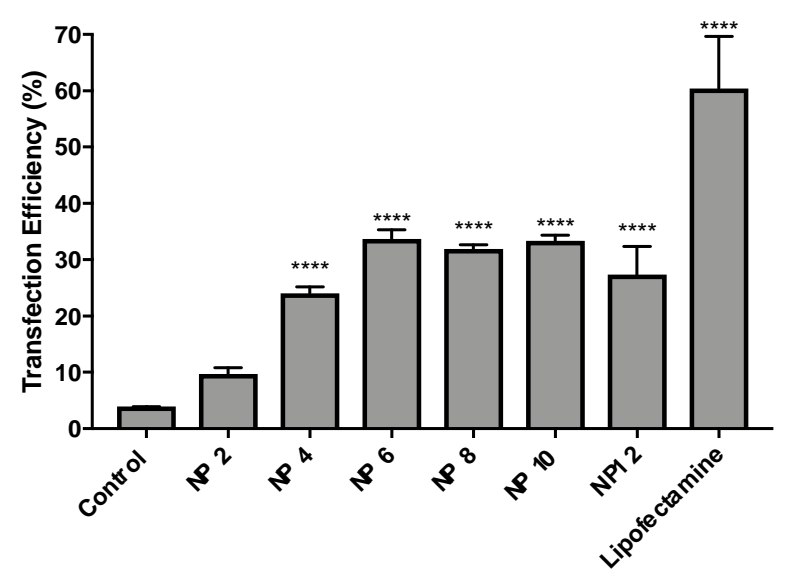

C
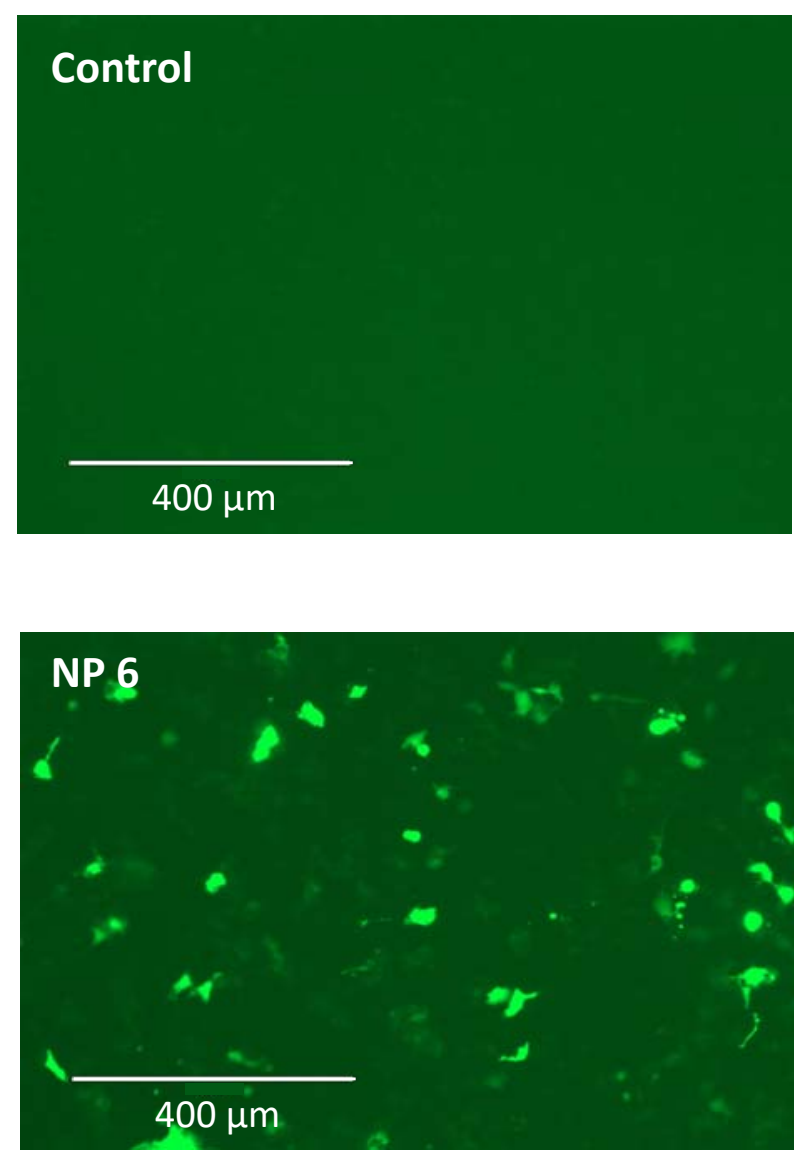

\section{B}

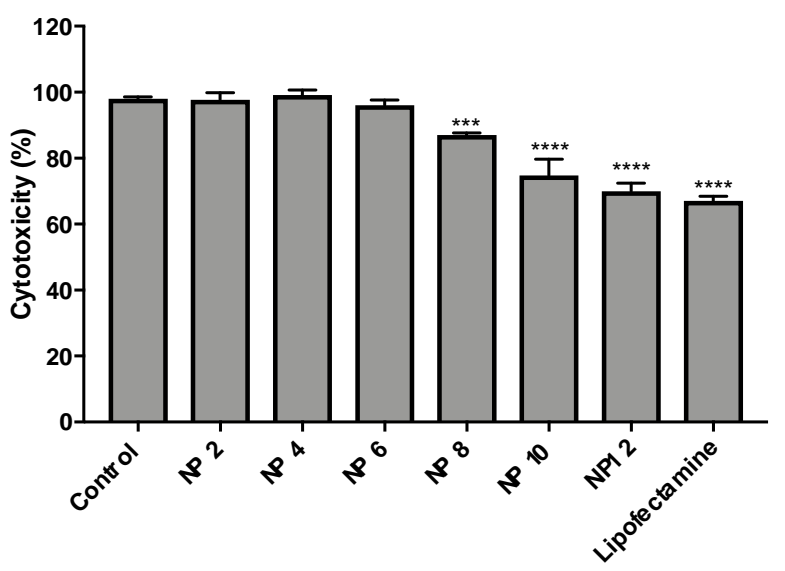

D (i)

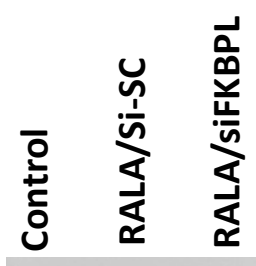

FKBPL (42 KDa)

\section{GAPDH (38 KDa)}

D (ii)

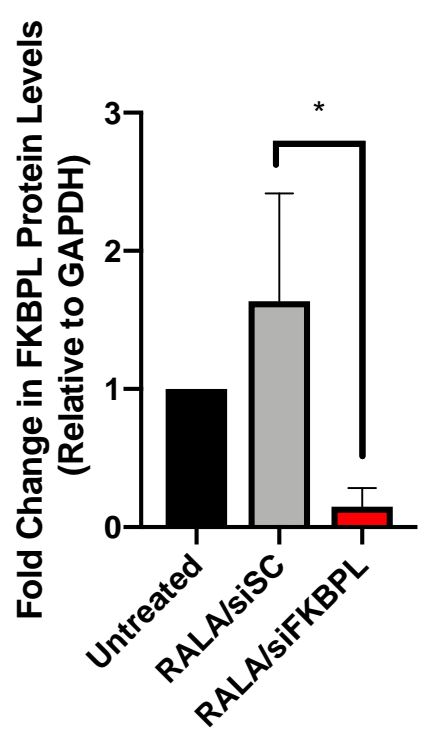

Figure 2: Transfection efficiency of nanoparticles comprising RALA/pEGFP-N1 and analysis of FKBPL protein levels after transfection with RALA/siFKBPL. (A) Cells were transfected for $4 \mathrm{~h}$ with nanoparticles at a range of $\mathrm{N}: \mathrm{P}$ ratios (0-10). Following transfection, the medium was replenished, and the plate incubated for $48 \mathrm{~h}$ before being fixed in $2 \%$ 
formaldehyde for quantification of transfection efficiency by flow cytometry. In a separate experiment, the medium was replaced following transfection, and samples incubated for $24 \mathrm{~h}$ before $10 \mu \mathrm{L}$ of MTS reagent was added directly to culture medium and samples incubated for a further $2 \mathrm{~h}$. Cell viability was calculated as a measure of fluorescence. (C) Images were taken at $48 \mathrm{~h}$ post-transfection to visualise the expression of the GFP (Scale bar= $200 \mu \mathrm{m}$ ). (D) Protein extracted from cell lysates was quantified, denatured in laemmli buffer, and subjected to SDS-PAGE gel electrophoresis. Protein was then transferred to a nitrocellulose membrane and blocked with 5\% skimmed milk. Bands of interest were detected using FKBPL primary antibody. Bands (untreated, RALA/siSC and RALA/siFKBPL) were visualised upon the addition of chemiluminescence using a G Box imaging system (FKBPL MW=42 kDa, GAPDH MW= $35.8 \mathrm{kDa})$. Images are representative of three independent repeats. Densitometry was quantified using ImageJ software (version 1.52a, NIH, USA). Data is reported as mean $\pm \mathrm{SEM}$, $\mathrm{n}=3$ and statistical significance calculated by means of one-way ANOVA with Dunnett posthoc tests for transfection and cell viability studies, and Tukey post-hoc tests for western blot analysis. 
A
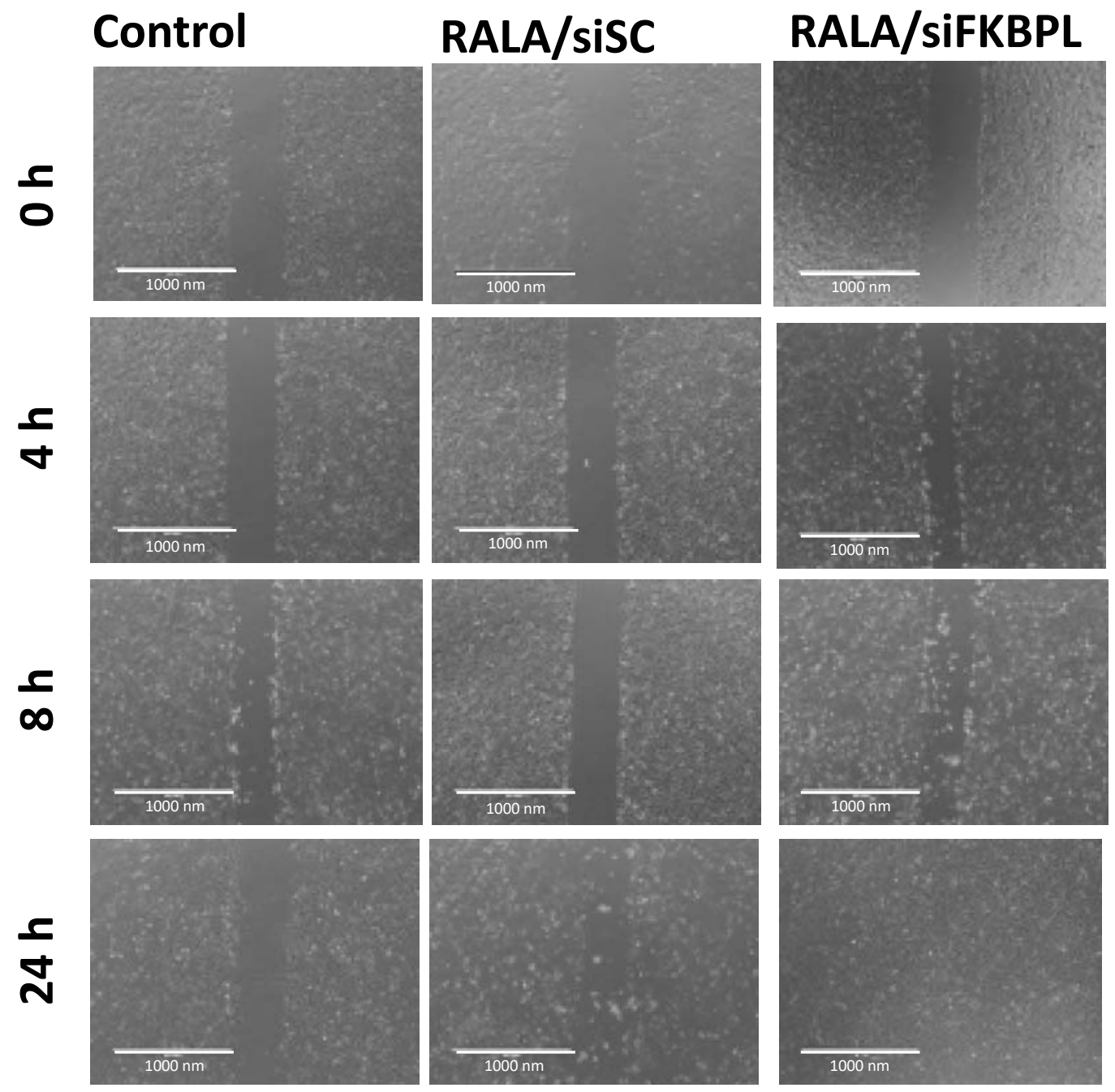

B

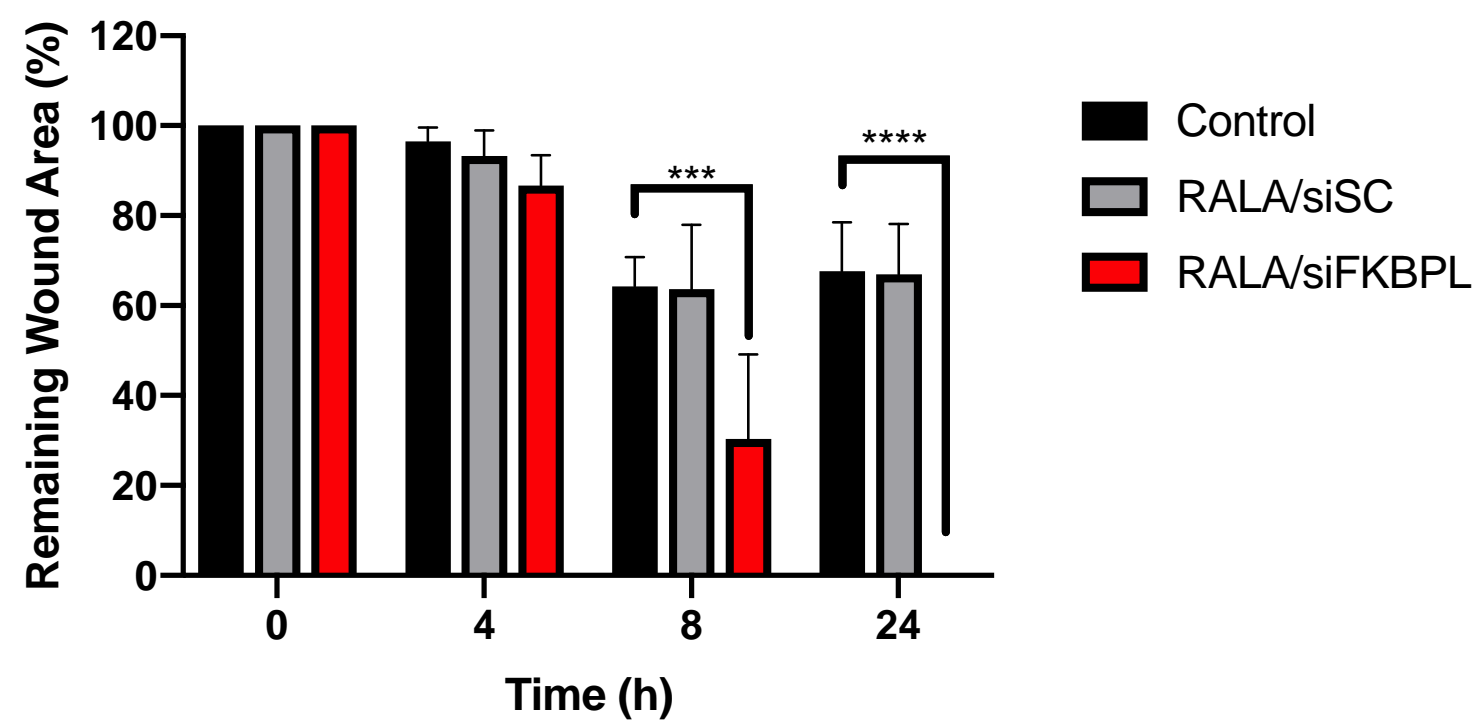


Figure 3: Wound scratch assay in HMEC-1 endothelial cells. Cells were transfected with RALA/siSC or RALA/siFKBPL in Opti-MEM in a 6-well plate and incubated at $37{ }^{\circ} \mathrm{C}$ in $5 \%$ $\mathrm{CO}_{2}$. Post-transfection, cells were transferred to wound scratch assay inserts (Ibidi, UK) and wounded the following day for migration monitoring. Images were analysed using ImageJ software (version 1.52a, NIH, USA). Images are representative of three independent repeats. $\mathrm{n}=3($ Scale bar $=200 \mu \mathrm{m})$ and statistical significance calculated by means of two-way ANOVA with Sidak post-hoc tests. 
A
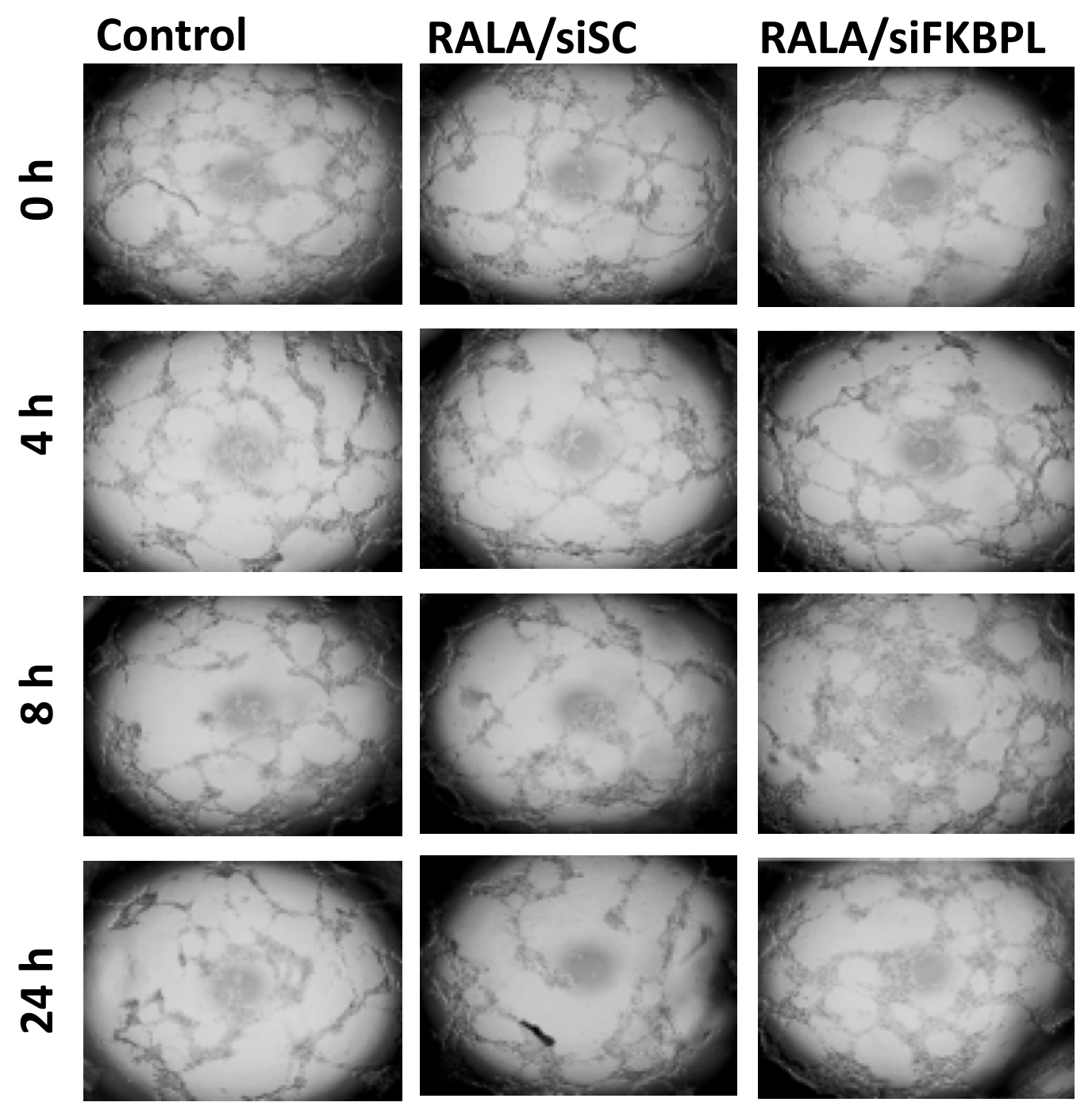

B

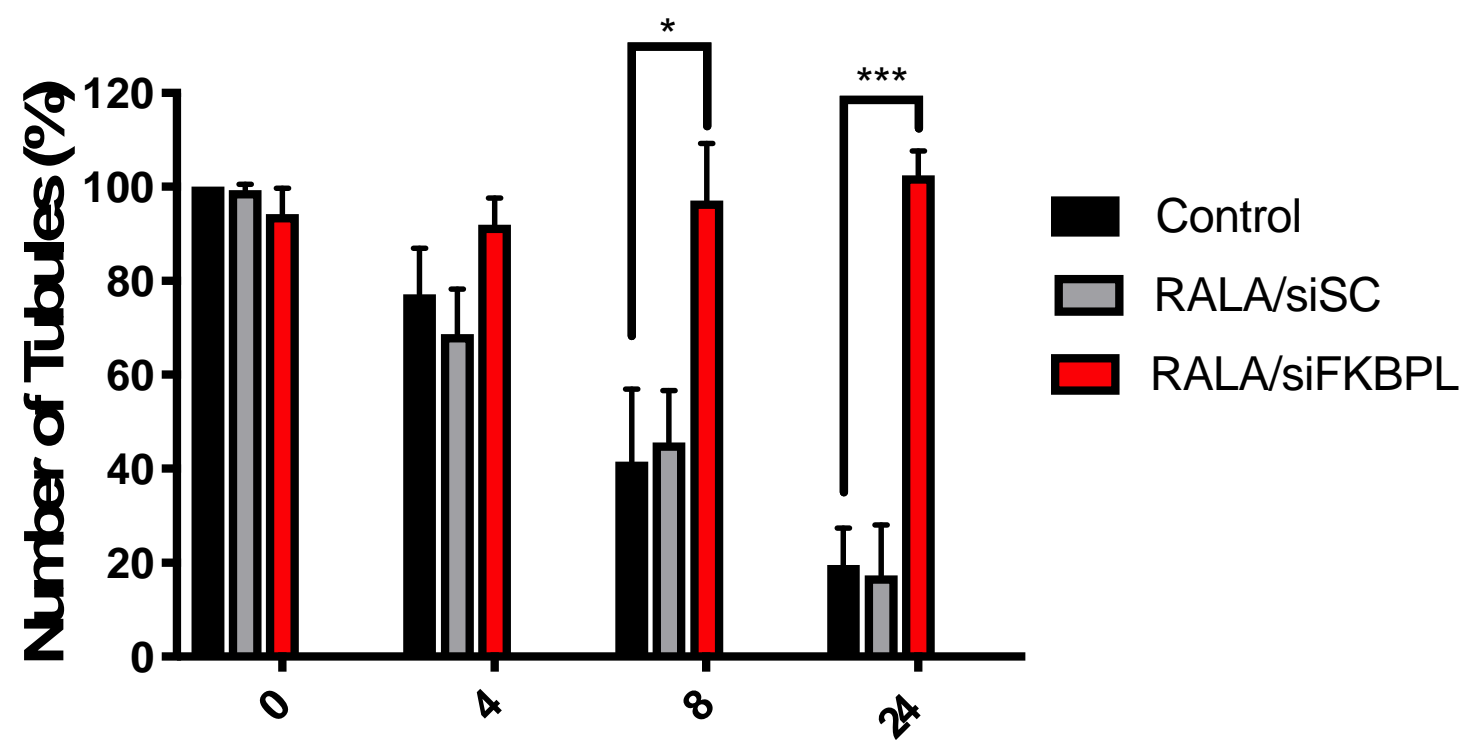


Figure 4: Effect of RALA/siFKBPL on Angiogenesis in HMEC-1 cells. Cells were transfected with $R A L A / s i S C$ or $R A L A / s i F K B P L$. At each time point, cells were re-seeded onto Matrigel in triplicate in 96 well plates at a density of 20,000 per well in serum-free conditions. Percentage tubule coverage was measured using the WimTube software package with microscope images taken at $4 \mathrm{x}$ magnification. Data is reported as mean $\pm \mathrm{SEM}, \mathrm{n}=3$. (Scale bar $=200 \mu \mathrm{m})$ and statistical significance calculated by means of two-way ANOVA with Dunnett post-hoc tests. 


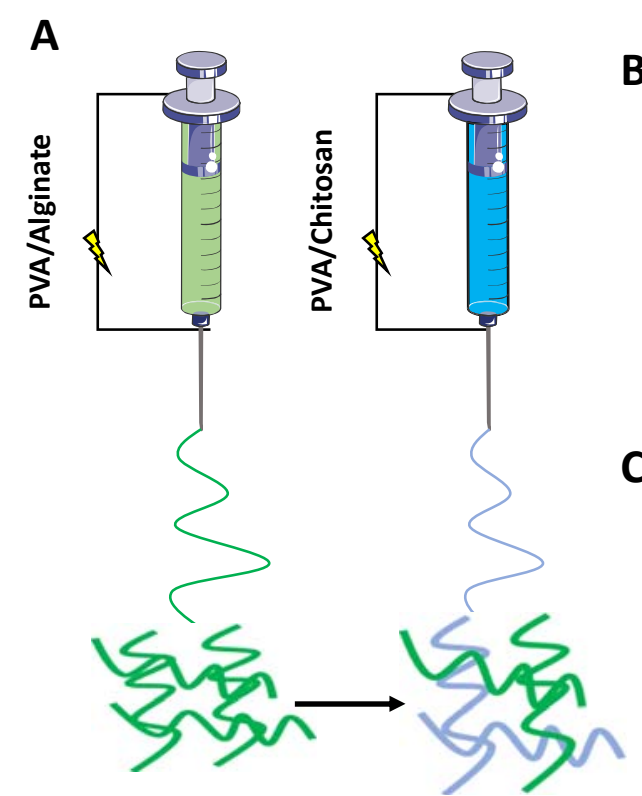

B

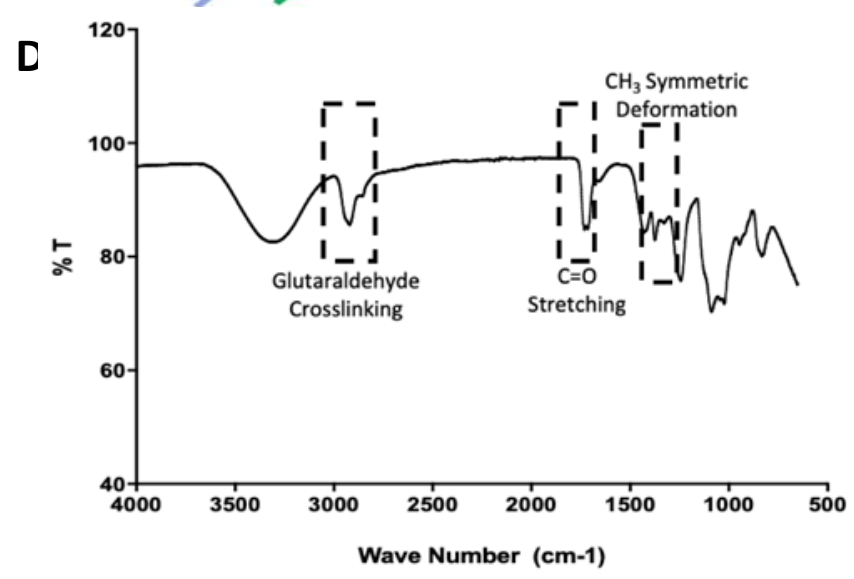

E
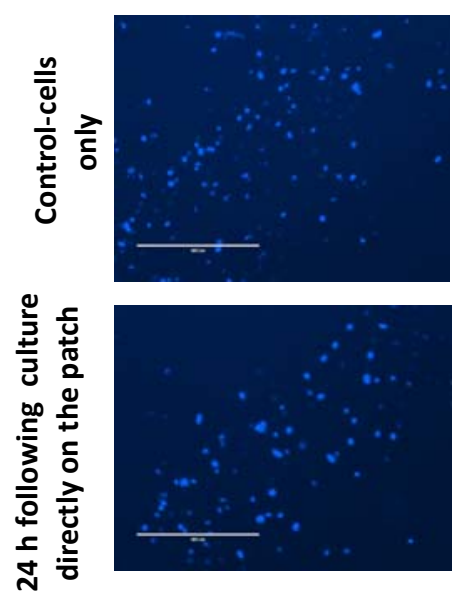

$\mathbf{F}$

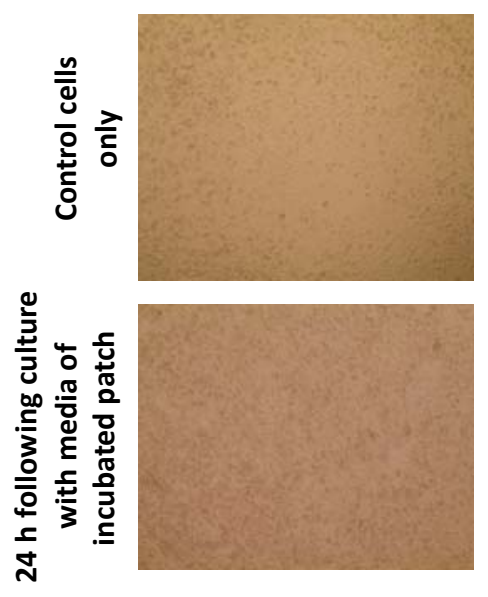

$24 \mathrm{~h}$ (1\% GA/methanol)

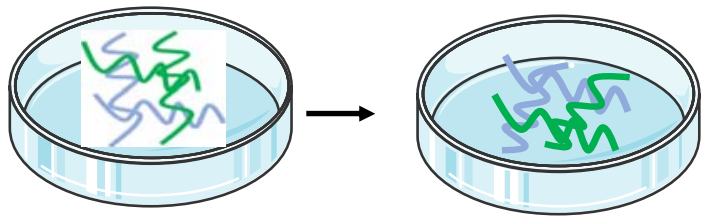

C

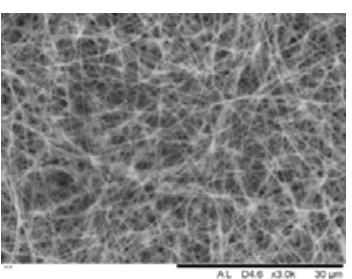

Pre-crosslinking

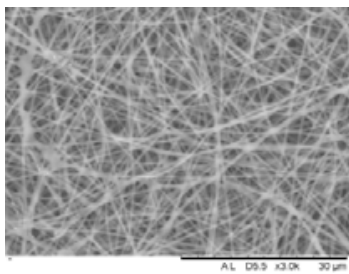

Post-crosslinking

Figure 5: Overview of the electrospinning and crosslinking process, and exhibition of the highly biocompatible nature of the nanofibres. Schematic representation of the bilayered ALG/PVA-CHT/PVA wound patch fabrication method (A) and crosslinking process with Glutaraldehyde (B). Representative SEM images (C) show the fibrous nature of the wound 
patch pre- and post-crosslinking. (D) FTIR analysis demonstrated that the crosslinking process was successful as at 2850 and $2750 \mathrm{~cm}-1 \mathrm{C}-\mathrm{H}$ stretching is evident and is related to aldehydes, a duplet absorption with peaks attributed to the alkyl chain. DAPI staining was used to measure proliferation of cells directly seeded onto the nanofibres (E). Cell viability was measured by indirect means (F) and quantitate analysis conducted, of which no significant (ns) effect was observed (G). Results are displayed as mean \pm SEM, $n=3$. (Scale bar $=30 \mu \mathrm{m}$ ) and statistical insignificance calculated by means of one-way ANOVA with Dunnett post-hoc tests. 
A

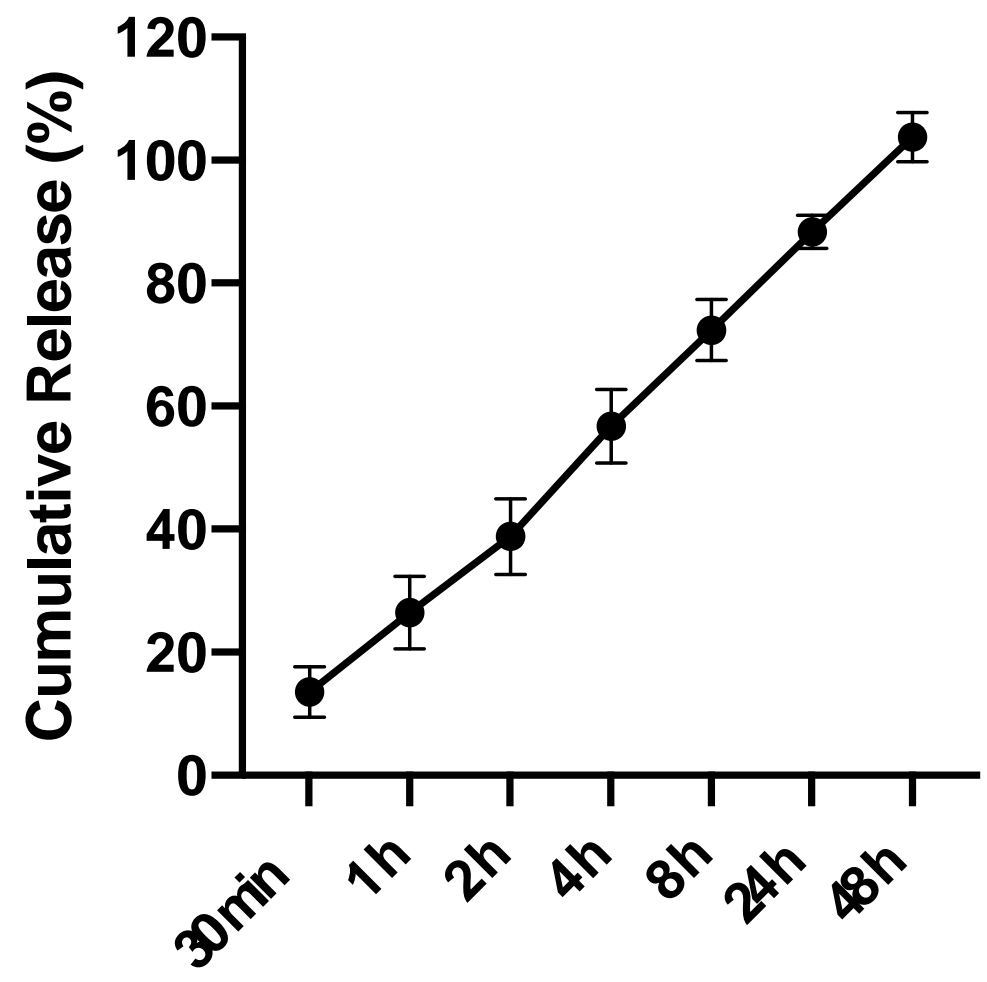

B

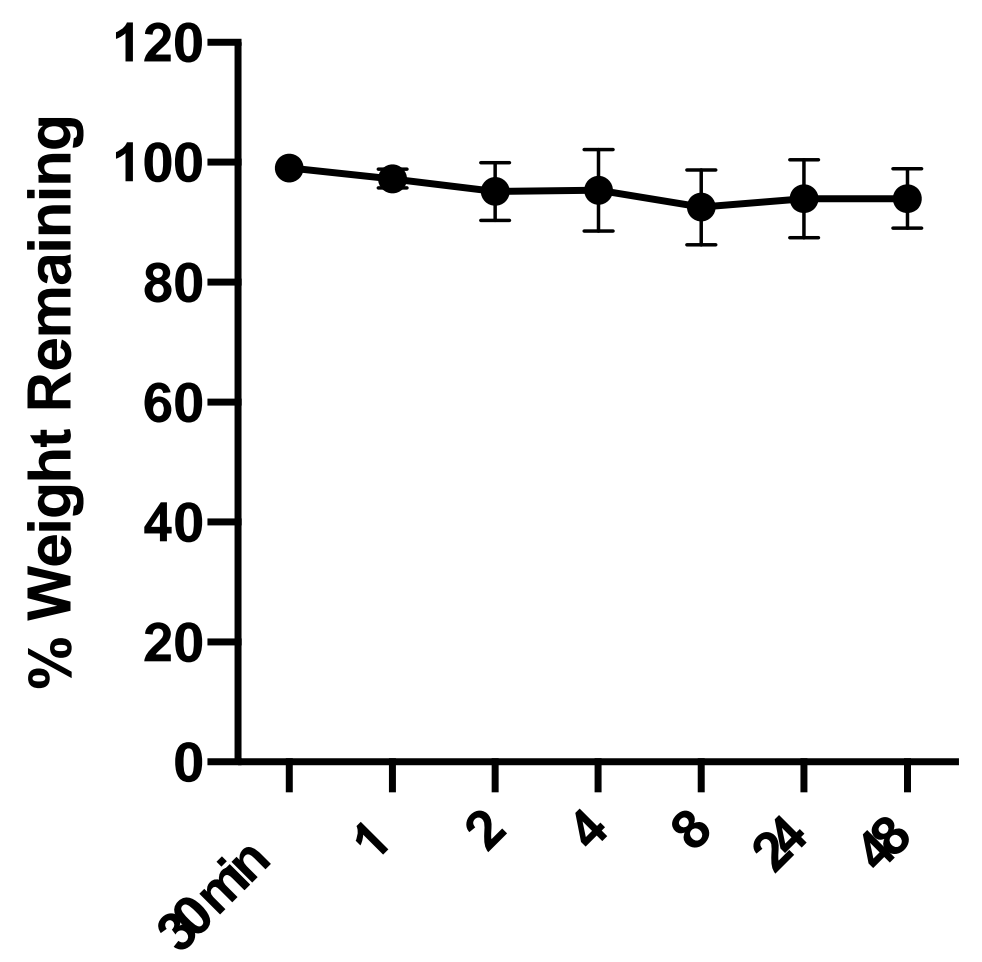

Figure 6: Nanoparticle release from bilayered wound patch and degradation analysis. (A)

Nanoparticle release as a function of time to $48 \mathrm{~h}$, from the crosslinked bilayered wound patch, loaded with $10 \mu \mathrm{g}$ of siFKBPL complexed with RALA. The wound patch was placed in $5 \mathrm{~mL}$ of ultrapure water, an aliquot of $200 \mu \mathrm{L}$ was taken at pre-defined time points. Samples were 
then treated with a proteinase-k enzyme to release the siFKBPL for quantification using the Picogreen assay. (B) Degradation profile of the crosslinked wound patch. Sections of the wound patch were cut to equal dimension and submerged in PBS solution at $37^{\circ} \mathrm{C}$ and then dried for $24 \mathrm{~h}$ before the dry weight was recorded. Results displayed as mean \pm SEM, $n=3$. 


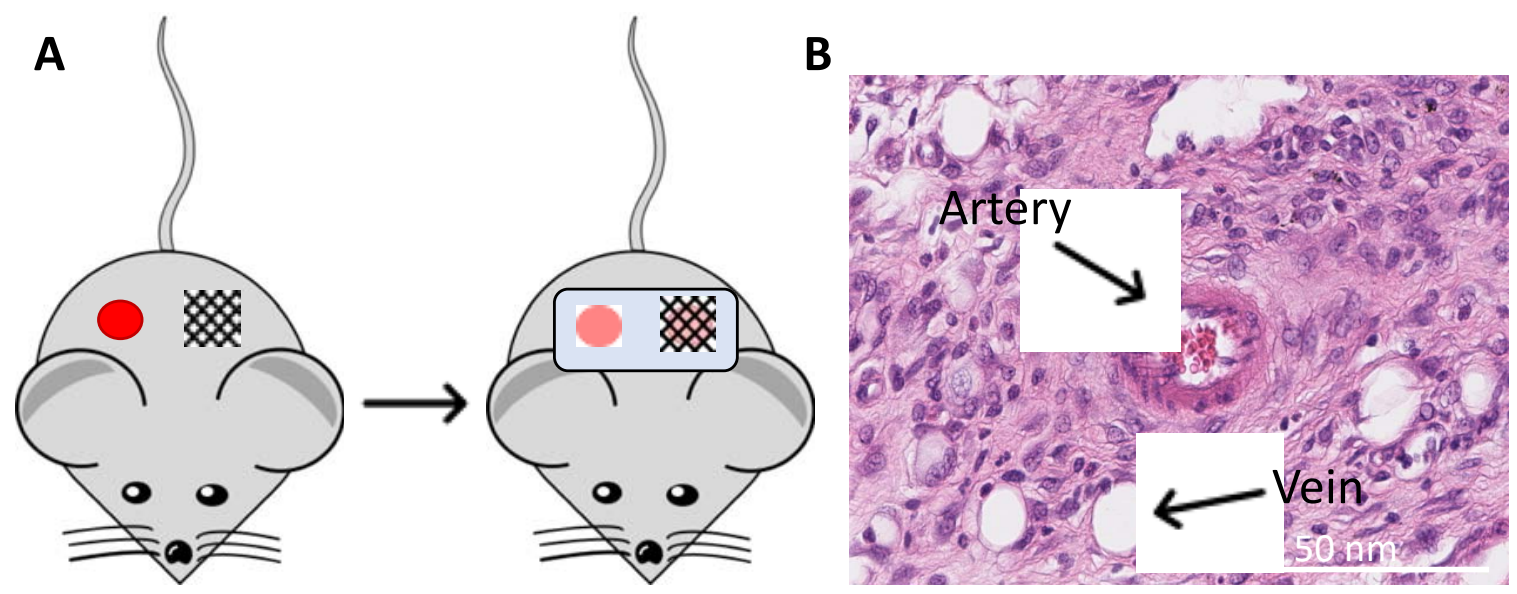

Wound Nanofibres $\square$ Film Dressing

C(i)

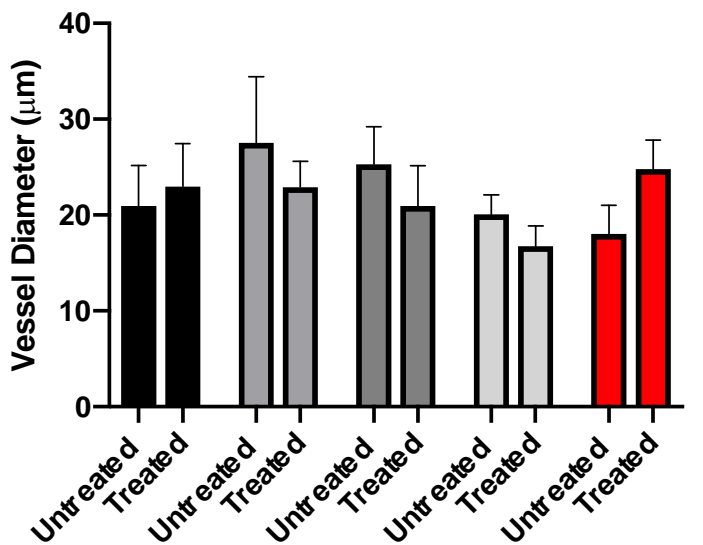

Unloaded Nanofibres

$\square$ RALA/siSC Nanofibres

$D(i)$

\section{Untreated}

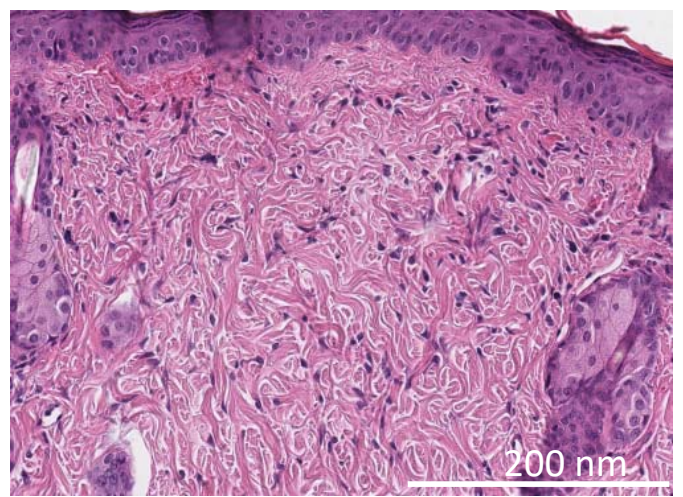

C(ii)

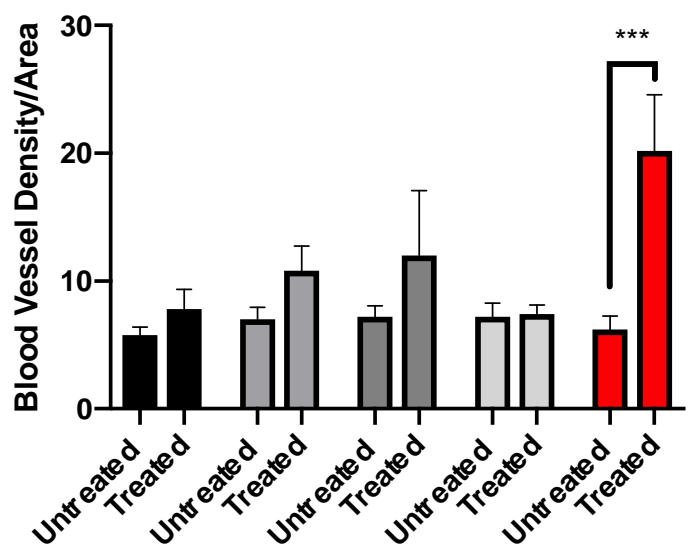

siFKBPL Nanofibres

Algisite $\square$ RALA/siFKBPL Nanofibres

D(ii)

RALA/siFKBPL

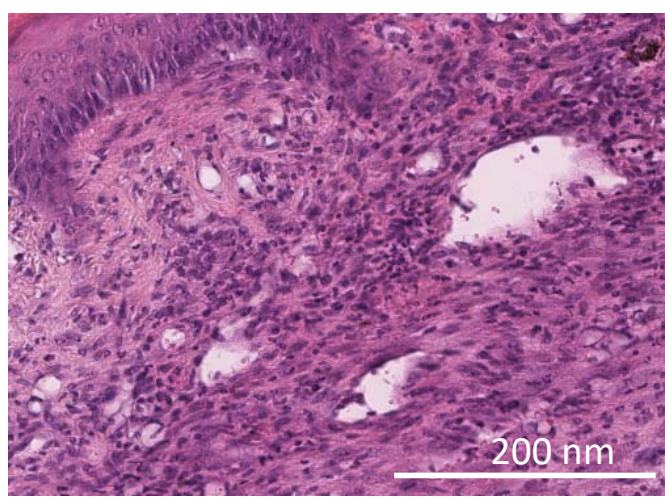

Figure 7: Figure showing the effects of treatments on vessel density and size in mice skin.

Each C57BL/6N mouse was euthanised at Day 7. The wound bed was then harvested and fixed in $10 \%$ formalin for $24 \mathrm{~h}$ prior to pathologic examination. Tissue sections were embedded in 
paraffin and then cut into sections of thickness $3 \mu \mathrm{m}$ and placed on glass microscope slides by the NI BioBank. Each sample was processed for hematoxylin and eosin (H\&E) staining. ImageJ analysis software (version 1.52a, NIH, USA) was then utilised for quantification of blood vessel density/measured area $\left(0.5 \mathrm{~mm}^{2}\right)$ and size for each of the treatment groups. (A) Schematic representation of the full-thickness wounding model used on C57BL/6J mice. (B) Representative image of blood vessels in the untreated skin of C57BL/6J mice. For each treatment group the vessel densities are represented in $(\mathrm{B}(\mathrm{I}))$, and vessel diameters are represented in $(\mathrm{B}(\mathrm{II}))$. ( $\mathrm{D}(\mathrm{i}-\mathrm{ii}))$ Representative images of untreated and RALA/siFKBPLbilayered wound patch treated mouse skin sections respectively. Data is reported as mean \pm $\mathrm{SEM}, \mathrm{n}=5$ and statistical significance calculated by means of two-way ANOVA with Sidak post-hoc tests (Images were taken at 20x magnification). 